\title{
Melatonin inhibits cytosolic mitochondrial DNA- induced neuroinflammatory signaling in accelerated aging and neurodegeneration
}

\author{
Abhishek Jauhari,, Sergei V. Baranov, ${ }^{1}$ Yalikun Suofu, ${ }^{1}$ Jinho Kim, ${ }^{1}$ Tanisha Singh, ${ }^{1}$ Svitlana Yablonska, ${ }^{1}$ Fang Li, ${ }^{1}$ Xiaomin Wang, ${ }^{1}$ \\ Patrick Oberly, ${ }^{2}$ M. Beth Minnigh, ${ }^{2}$ Samuel M. Poloyac, ${ }^{2}$ Diane L. Carlisle, ${ }^{1}$ and Robert M. Friedlander ${ }^{1}$
}

'Department of Neurological Surgery and 2Department of Pharmaceutical Sciences, University of Pittsburgh, Pittsburgh, Pennsylvania, USA

\begin{abstract}
Chronic inflammation is a pathologic feature of neurodegeneration and aging; however, the mechanism regulating this process is not understood. Melatonin, an endogenous free radical scavenger synthesized by neuronal mitochondria, decreases with aging and neurodegeneration. We proposed that insufficient melatonin levels impair mitochondrial homeostasis, resulting in mitochondrial DNA (mtDNA) release and activation of cytosolic DNA-mediated inflammatory response in neurons. We found increased mitochondrial oxidative stress and decreased mitochondrial membrane potential, with higher mtDNA release in brain and primary cerebro-cortical neurons of melatonin-deficient aralkylamine $\mathrm{N}$-acetyltransferase (AANAT) knockout mice. Cytosolic mtDNA activated the cGAS/STING/IRF3 pathway, stimulating inflammatory cytokine generation. We found that Huntington's disease mice had increased mtDNA release, cCAS activation, and inflammation, all inhibited by exogenous melatonin. Thus, we demonstrated that cytosolic mtDNA activated the inflammatory response in aging and neurodegeneration, a process modulated by melatonin. Furthermore, our data suggest that AANAT knockout mice are a model of accelerated aging.
\end{abstract}

\section{Introduction}

Mitochondrial dysfunction is a common feature of neurodegeneration. Neuronal mitochondrial DNA (mtDNA) damage during neurodegeneration results from reactive oxygen species-initiated (ROS-initiated) oxidation and impairment of DNA repair (1-7). Decreased mitochondrial membrane potential (MMP) and increased mitochondrial permeability regulate release of mtDNA into the cytosol $(8,9)$ followed by cyclic guanosine monophosphate-adenosine monophosphate synthase (cGAS) pathway activation. cGAS senses cytosolic DNA including self or foreign DNA (10, 11). cGAS activates innate immune responses through production of cyclic GMP-AMP which binds and activates STING (12-14). STING activates IRF3 and NF- $\mathrm{KB}$, which induce interferon (IFN) and proinflammatory interleukin expression (15). Mitochondrial ROS induces caspase- 1 activation and secretion of proinflammatory cytokines (IL-1 $\beta$ and IL-18) (16). These mechanisms are key regulators of neurodegeneration since synaptic pruning, which underlies progressive neurological decline in aging and neurodegenerative diseases along with neuronal death (17) is mediated by cytokine activated complement and microglia (18). Furthermore, caspase-1 inhibition is neuroprotective in acute and chronic neurodegeneration (19-25).

Huntington's disease (HD) is caused by mutant huntingtin (mHTT) (26), a protein with an expanded polyglutamine region which binds to the TIM23 mitochondrial protein import complex

Conflict of interest: RMF is on the board of NeuBase Therapeutics. Copyright: () 2020, American Society for Clinical Investigation. Submitted: November 15, 2019; Accepted: March 5, 2020; Published: May 11, 2020. Reference information: J Clin Invest. 2020;130(6):3124-3136. https://doi.org/10.1172/JCl135026. and inhibits protein import $(27,28)$. Impaired mitochondrial protein import increased mitochondrial oxidative stress. Striatum and cortex exhibited 8-fold more mtDNA damage than nuclear DNA damage, suggesting that mtDNA is more susceptible to oxidative damage than nuclear DNA in HD (4).

Melatonin, a neuroprotective hormone, is a potent free radical scavenger (29-33) that offers protection from mHTT-mediated neurotoxicity (34). In neurons, melatonin is exclusively synthesized in the mitochondrial matrix (35), where it prevents ROS accumulation $(36,37)$. Melatonin protects neurons by ameliorating loss of MMP, cytochrome c release, caspase activation, and proinflammatory cytokine activation during neurodegeneration $(30,36$, 38-43). Melatonin progressively decreases with age and in neurodegenerative diseases including HD, Parkinson's disease, and Alzheimer's disease $(44,45)$. Melatonin loss likely results in enhanced neuronal vulnerability associated with aging and disease.

We report the first demonstration of cytosolic mtDNA release in neurons during neurodegeneration and place mitochondrial dysfunction as an initiating event resulting in pathologic inflammation and caspase activation. Furthermore, we demonstrate the direct role of melatonin in modulating cytosolic mtDNA release in an accelerated aging model, and in an HD model of neurodegeneration. We demonstrate that both melatonin deficiency in normal cells and the presence of mHTT in a mouse model of neurodegeneration increases ROS damage, mtDNA release, activation of the cGAS pathway, and pathologic inflammatory response, leading to synaptic loss and neurodegeneration. Our data therefore demonstrate that melatonin deficits modulate neuronal and synaptic vulnerability, and points to melatonin as a potential therapy for age-related ailments. 
A

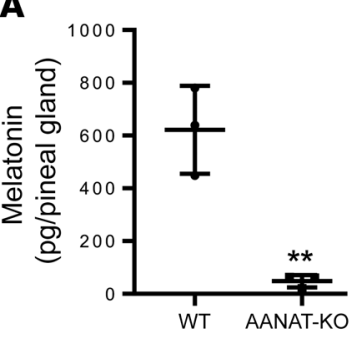

B

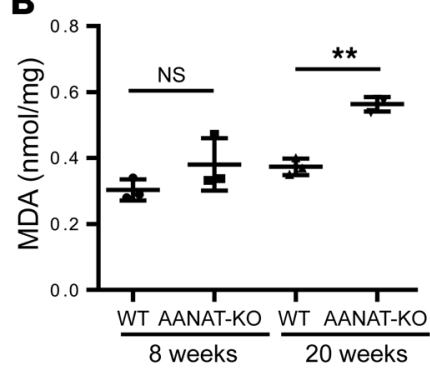

E

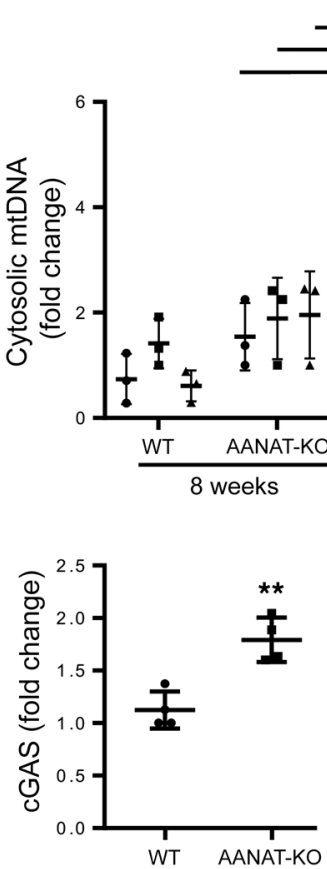

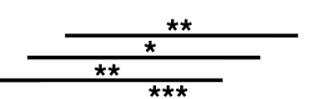
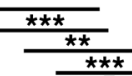

- mt-Dloop1 - $\mathrm{mt}-\mathrm{CO} 1$ A mt-Dloop3
C

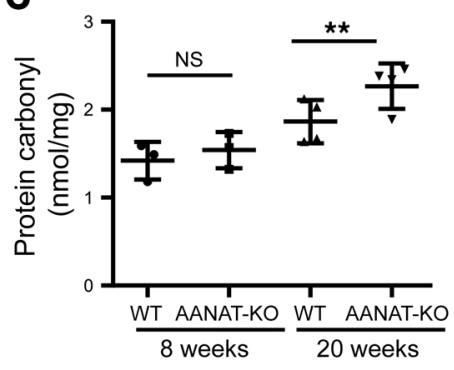

D

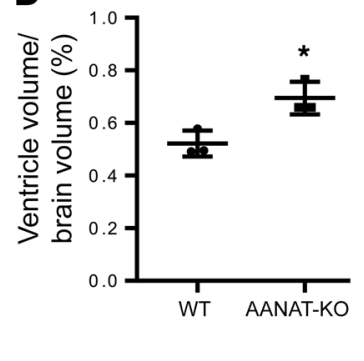

$\mathbf{F}$

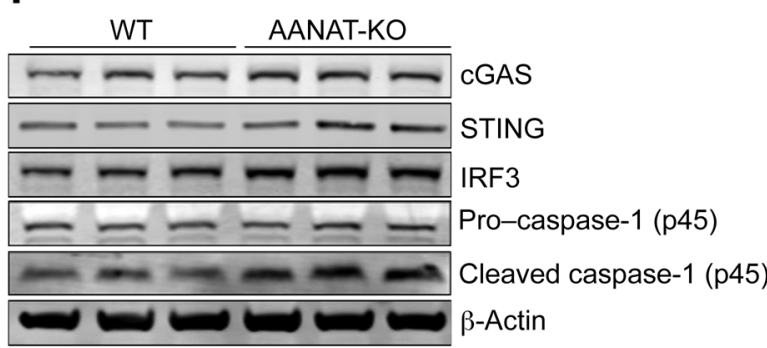

H
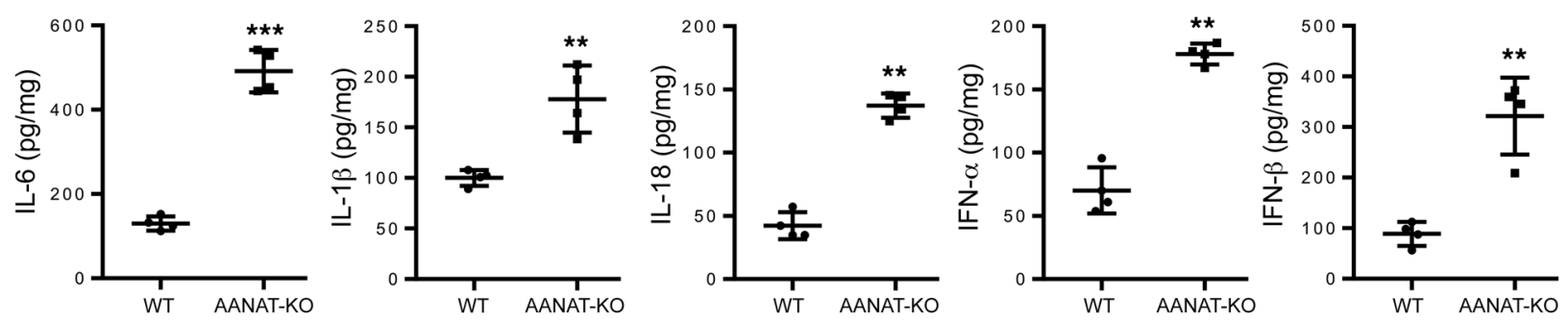

Figure 1. Melatonin deficiency-induced mtDNA release mediated neuroinflammation. (A) LC-MS quantification of melatonin in pineal gland isolated at 4:00 a.m. from 20-week-old WT and AANAT-KO mice $(n=3)$. (B) Quantification of malondialdehyde (MDA), an indicator of lipid peroxidation, and (C) protein carbonylation, an indicator of protein oxidation in whole brain of 8- and 20-week-old WT and AANAT-KO mouse brain lysate ( $n=3$ ). (D) Ventricle volume in 20-week-old WT and AANAT-KO brain $(n=3)$. (E) qPCR of cytosolic mtDNA in 8- and 20-week-old WT and AANAT-KO brain using primers for $\mathrm{mt}-\mathrm{CO} 1$, mt-Dloop1, and mt-Dloop3, mitochondrial genes. Cytosolic mtDNA is plotted relative to amount of WT brain after normalization to $\beta$-actin from the corresponding total DNA lysate, $n=3$ for 8 weeks, $n=4$ for 20 weeks. (F) Representative immunoblots and (G) quantitation for cGAS, STING, IRF3, caspase-1, and $\beta$-actin in WT and AANAT-KO brain lysates. $\beta$-actin was used as a loading control; data are expressed as relative to WT control $(n=4)$. (H) Cytokine ELISA in brain lysate of 20-week-old WT and AANAT-KO mice expressed as pg of cytokine per mg of protein lysate $(n=4)$. All data are expressed as mean \pm SD, analyzed by Student's $t$ test $(\mathbf{A}, \mathbf{D}, \mathbf{G}$, and $\mathbf{H})$, or ANOVA followed by Tukey's test (B, C, and $\mathbf{E}) .{ }^{*} P<0.05 ;{ }^{* *} P<0.01 ;{ }^{* * *} P<0.001$.

\section{Results}

AANAT deletion results in mtDNA-mediated inflammation. Melatonin is a neuroprotective hormone synthesized in neuronal mitochondria $(30,35)$. To explore the role of melatonin in the brain, we created aralkylamine $\mathrm{N}$-acetyltransferase ${ }^{-/-}$(AANAT-KO) mice in a CBA/J genetic background, a strain that produces melatonin (46, 47). AANAT is the penultimate enzyme in melatonin synthesis, and is located in the mitochondrial matrix $(35,48)$. Melatonin concentration measured in pineal during peak production (4 a.m.) is reduced to background in AANAT-KO mice as compared with WT mice (Figure 1A), demonstrating effective knockout of melatonin synthesis. At 20 weeks of age, AANAT-KO mice gained less weight than WT mice, a difference not found in 8-week-old mice (Supplemental Figure 1A; supplemental material available online with this 
article; https://doi.org/10.1172/JCI135026DS1). Melatonin is an antioxidant, therefore we studied protein carbonylation and lipid peroxidation, markers of oxidative damage associated with aging and neurodegeneration, in brains of AANAT-KO mice $(49,50)$. AANAT-KO brain had higher levels of protein carbonylation and lipid peroxidation (Figure 1, B and C), suggesting that melatonin absence results in increased cerebral oxidative stress. Increased ventricle size is associated with aging (51), and in 20-week-old AANAT-KO mice we also found a $25 \%$ increased corrected ventricle size $(P<0.05)$ in the context of a smaller brain $(10 \%$ not significant), therefore representing atrophy and not hydrocephalus (Figure 1D and Supplemental Figure 1, B and C). The combination of premature neurodegeneration, weight loss, increased ROS damage, and neuroinflammation suggests AANAT-KO mice as a model of accelerated aging.

Given the role of melatonin in mitochondrial homeostasis, we evaluated whether AANAT-KO mice have elevated levels of cytosolic mtDNA. By 20 weeks but not at 8 weeks of age, we identify a 3.6 -fold \pm 0.8 -fold increase of cytosolic mtDNA in AANAT-KO as compared with WT mice (Figure 1E). Release of mtDNA into the cytosol activates an inflammatory response via the cGAS/STING/IRF3 signaling axis (14). We found elevated levels of cGAS/STING/IRF3 proteins in 20-week-old AANATKO brains compared with controls (Figure 1, F and G). qRT-PCR data (Supplemental Figure 1D), which demonstrated increased mRNA expression at 20 weeks but not at 8 weeks of age, complemented these results. Moreover, caspase- 1 activation increased in AANAT-KO brains (Figure 1, F and G). Caspase-1 activation along with increased cGAS, STING, and IRF3 expression in AANAT-KO mouse brain indicates activation of a neuroinflammatory response (52), and neuronal caspase- 1 is a driver of neurodegeneration in vivo (21-24, 31, 52). Furthermore, AANAT-KO brain has elevated levels of proinflammatory cytokines and their respective mRNA (IL-6, IL-18, IL-1 $\beta$, IFN- $\alpha$, and IFN- $\beta$ ) compared with controls (Figure $1 \mathrm{H}$ and Supplemental Figure $1 \mathrm{E}$ ). This data demonstrates that melatonin deficit results in accelerated age-related elevated ROS damage, release of mtDNA activating the cGAS pathway, and a proinflammatory response. In light of these results, progressive melatonin reduction associated with human aging and in neurodegenerative disease likely plays a role in increasing physiologic and pathologic degeneration.

Melatonin mitigates mtDNA-mediated inflammatory response in AANAT-KO primary cerebrocortical neurons. To complement in vivo studies with neuron-specific data, AANAT-KO and WT primary cerebrocortical neurons (PCNs) cultured for 21 days were assayed for MMP and mitochondrial ROS. MMP decreased and ROS increased in AANAT-KO PCNs; exogenous melatonin administration restored both parameters (Figure 2A and Supplemental Figure 2A). MMP exists as a gradient in neurons, whereby synaptic mitochondria have lower MMP than somal mitochondria, resulting in synaptic vulnerability (53). MMP is similar in somal mitochondria ( $<10 \mu \mathrm{m}$ from nucleus) but decreased in synaptic AANAT-KO mitochondria ( $>50 \mu \mathrm{m}$ from nucleus) (Supplemental Figure $2 \mathrm{~B}$ ). Thus, the MMP gradient is exacerbated in AANAT-KO PCNs, indicating greater synaptic vulnerability. Like AANAT-KO brain, AANAT-KO PCNs demonstrate increased cytosolic mtDNA, cGAS/STING/ IRF3 levels, caspase-1 activation, and proinflammatory cytokine expression, all of which were inhibited by melatonin (Figure 2, $\mathrm{B}-\mathrm{E})$. Consistent with in vivo data, neuronal melatonin depletion results in mitochondrial ROS damage, mtDNA release, and elevation of inflammatory cytokines, all inhibited by melatonin.

Neuroinflammation and neurodegeneration are increased in differentiated N2a AANAT-KO cells, and their effects are attenuated by melatonin. To advance our mechanistic investigation of AANATKO effects on neurons, we generated N2a AANAT-KO neuroblastoma cells (Supplemental Figure 3A). We confirmed that N2a cells produce melatonin, and confirmed its absence in AANATKO cells (Supplemental Figure 3, B and C). After differentiation into mature neurons, WT and AANAT-KO N2a cells were assayed for mtDNA oxidative damage by immunofluorescence using an antibody against 8-hydroxyguanasine (8-OHDG). AANAT-KOdifferentiated neurons have increased mtDNA damage, slower mitochondrial protein import, lower MMP, and higher ROS as compared with WT-differentiated neurons. Abnormalities are mitigated by melatonin (Supplemental Figure 3, D-G). Further, mitochondrial permeability transition was higher in mitochondria of AANAT-KO-differentiated neurons and melatonin partially inhibited it (Supplemental Figure 3H). Mitochondrial damage and dysfunction in AANAT-KO cells, which are rescued by melatonin, suggest a critical role for melatonin in mitochondrial health. Interestingly, melatonin deficiency in AANAT-KO N2a cells only resulted in mtDNA-mediated inflammatory response in differentiated and not in undifferentiated AANAT-KO N2a cells (data not shown), suggesting that postmitotic cells are dependent on melatonin but undifferentiated cells are not. This may be explained by either greater vulnerability of postmitotic neuronal mitochondrial to oxidative stress or the fact that differentiated cells are more dependent on mitochondrial respiration compared with undifferentiated/dividing cells (54), and thus inefficient mitochondrial function is more disruptive.

Consistent with AANAT-KO PCNs, mtDNA release was increased in differentiated AANAT-KO N2a neurons, and the increase was prevented by melatonin (Figure 3A). We then evaluated 3 common regulators of inflammatory responses: $\mathrm{NF}-\kappa \mathrm{B}$, caspase-1, and cGAS/STING/IRF3. NF- $\kappa \mathrm{B}$ was phosphorylated and caspase- 1 was cleaved, suggesting activation of both pathways, whereas cGAS/STING/IRF3 levels increased in AANAT-KO-differentiated N2a neurons. Melatonin administration inhibited all inflammatory signaling pathways in AANAT-KO-differentiated neurons (Figure 3, B and C). Consistent with this data, AANAT-KO N2a neurons secrete elevated levels of proinflammatory cytokines, all of which were inhibited by melatonin (Figure 3D). As an additional indicator of cellular stress, caspase-3 activation increased in AANAT-KO-differentiated N2a neurons, an effect inhibited by melatonin (Supplemental Figure 4, A and B). Inflammatory signaling modulates synaptic pruning (55). Synaptic and neuritic degeneration were prominent in AANAT-KO-differentiated N2a neurons as compared with WT neurons. AANAT-KO-differentiated neurons had reduced average neurite length and number, both of which were rescued by exogenous melatonin (Figure 3, E-G). Expression of PSD95, a postsynaptic marker, and synaptophysin, a presynaptic marker, were reduced in AANAT-KO-differentiated neurons, suggesting synaptic degeneration. Melatonin increased the levels of these markers (Supplemental Figure 4, A and B). 

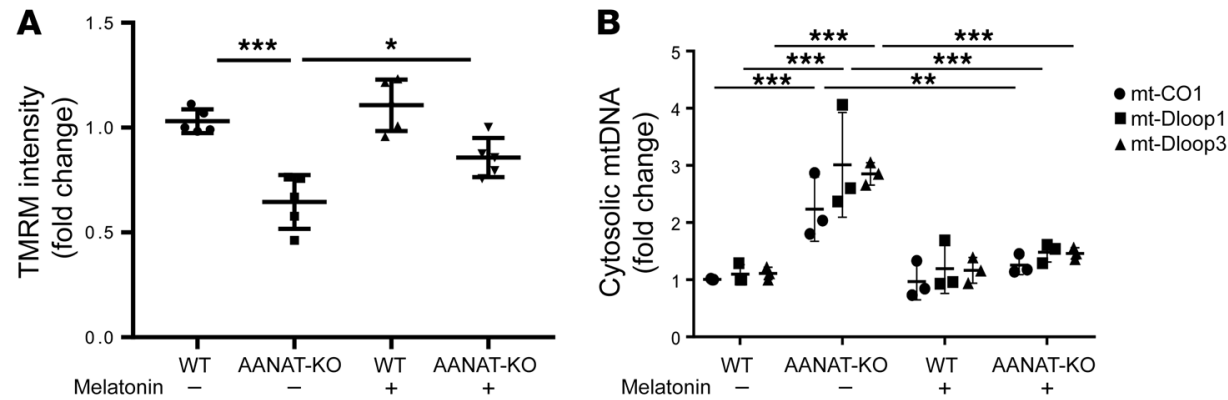

C

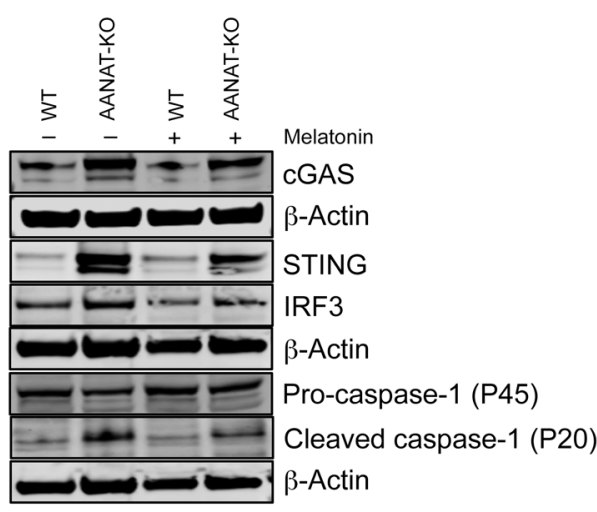

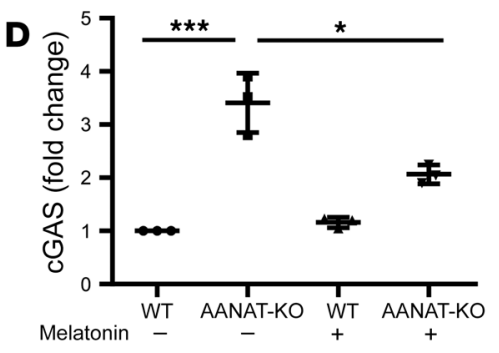

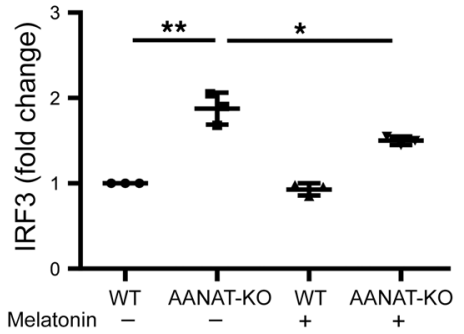

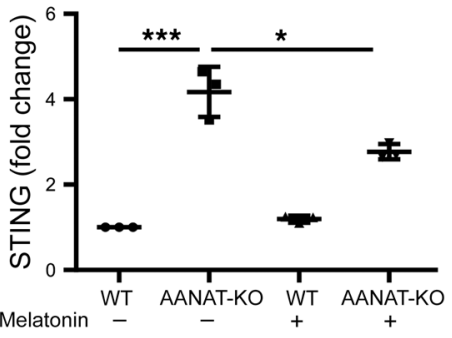

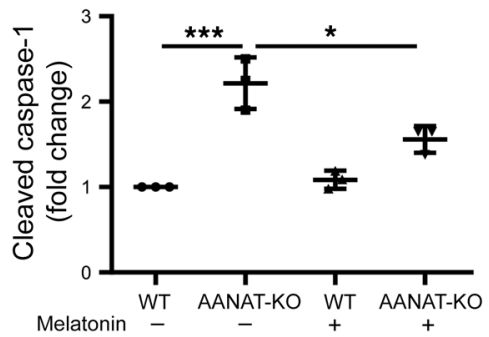

E
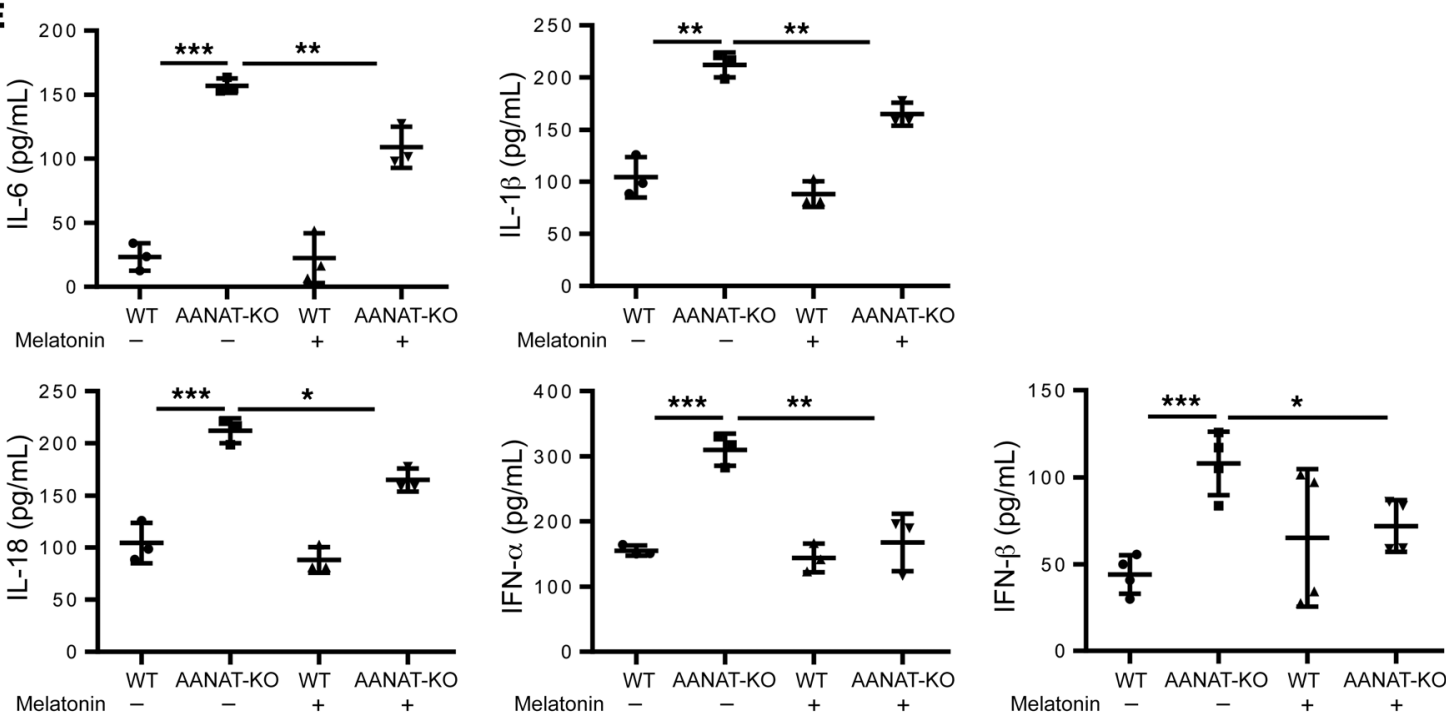

Figure 2. AANAT-KO induces mtDNA release and subsequent inflammation in PCNs. (A) Analysis of MMP by TMRM in WT and AANAT-KO PCNs at DIV21 (21 days in vitro) with or without melatonin $(5 \mu \mathrm{M})$ in culture medium. TMRM fluorescence is plotted relative to WT PCN after normalization to nuclear stain $(n=5)$. (B) qPCR analysis of cytosolic mtDNA in WT and AANAT-KO PCNs using primers for mt-C01, mt-Dloop1, and mt-Dloop3. Cytosolic mtDNA is plotted relative to amount of WT PCN after normalization to $\beta$-actin from the corresponding total DNA lysate $(n=3)$. (C) Representative immunoblot and (D) quantitation for CGAS, STING, IRF3, caspase-1, and $\beta$-actin in WT and AANAT-KO. $\beta$-actin was a loading control and data are expressed relative to untreated WT $(n=3)$. (E) Cytokine ELISA in culture medium of WT and AANAT-KO PCNs expressed as pg cytokine per mL of culture medium. PCNs grown with or without $5 \mu \mathrm{M}$ melatonin $(n=3)$. All experiments were performed at DIV21, expressed as the mean \pm SD, analyzed by ANOVA followed by Tukey's test. ${ }^{*} P<0.05$; ${ }^{*} P<0.01$; ${ }^{* *} P<0.001$. 


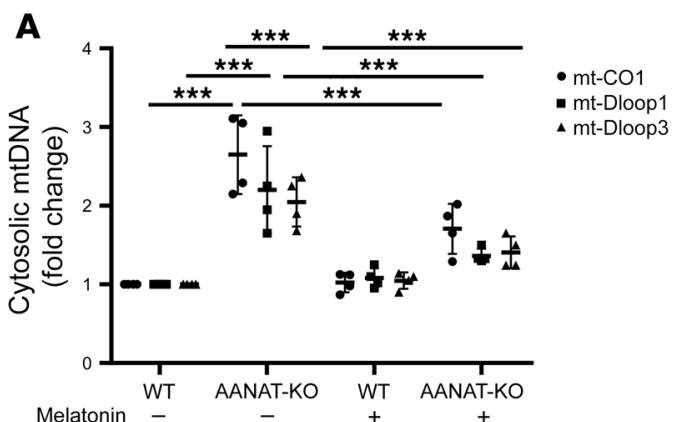

B

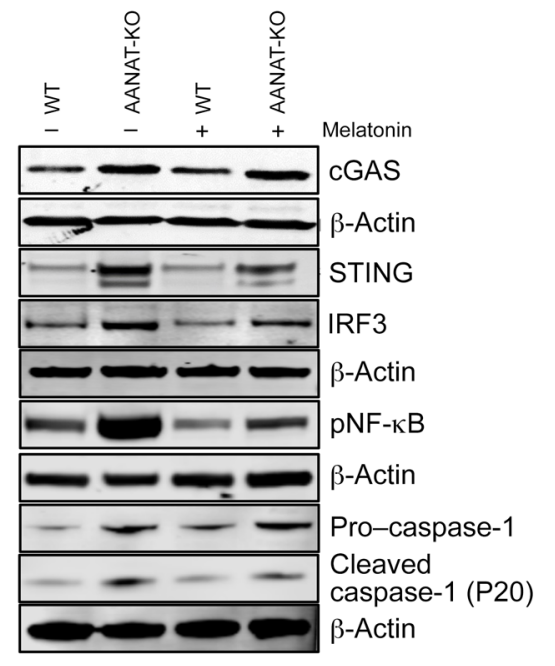

C
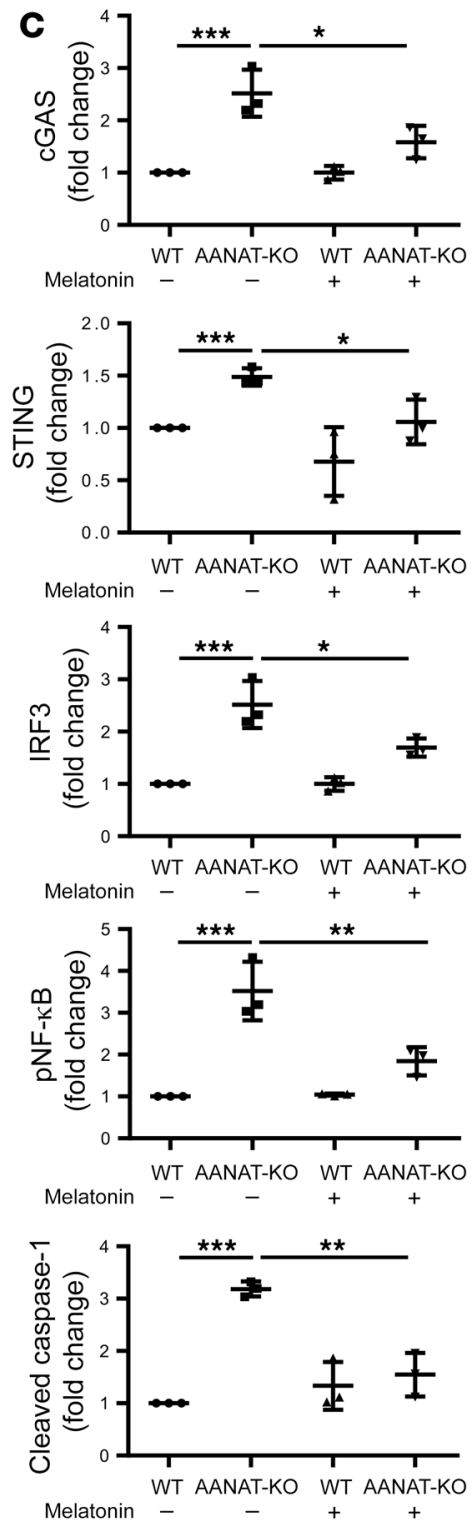

D
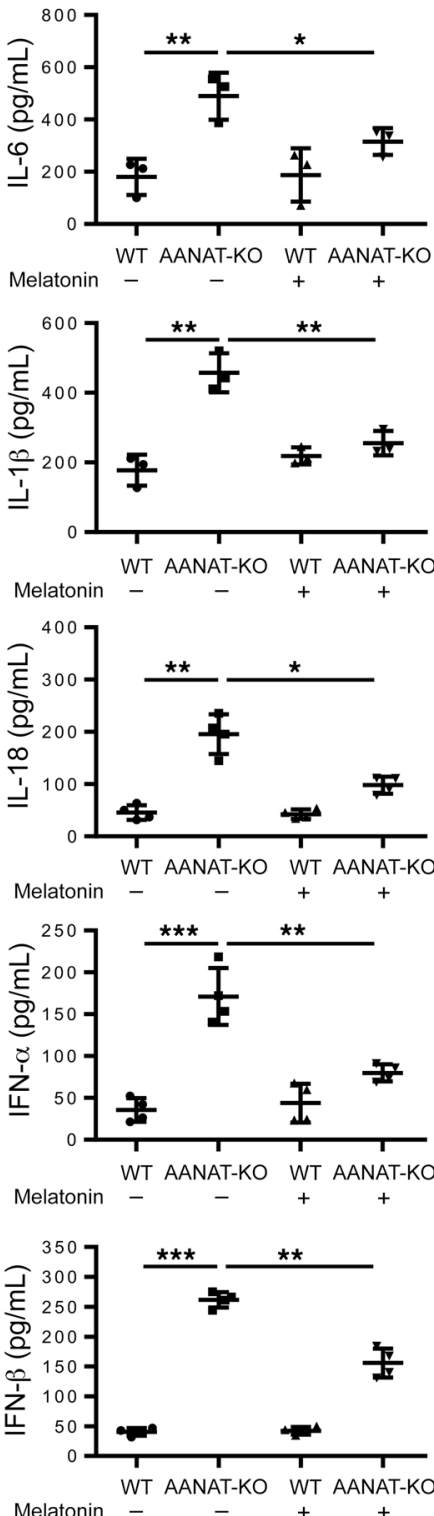

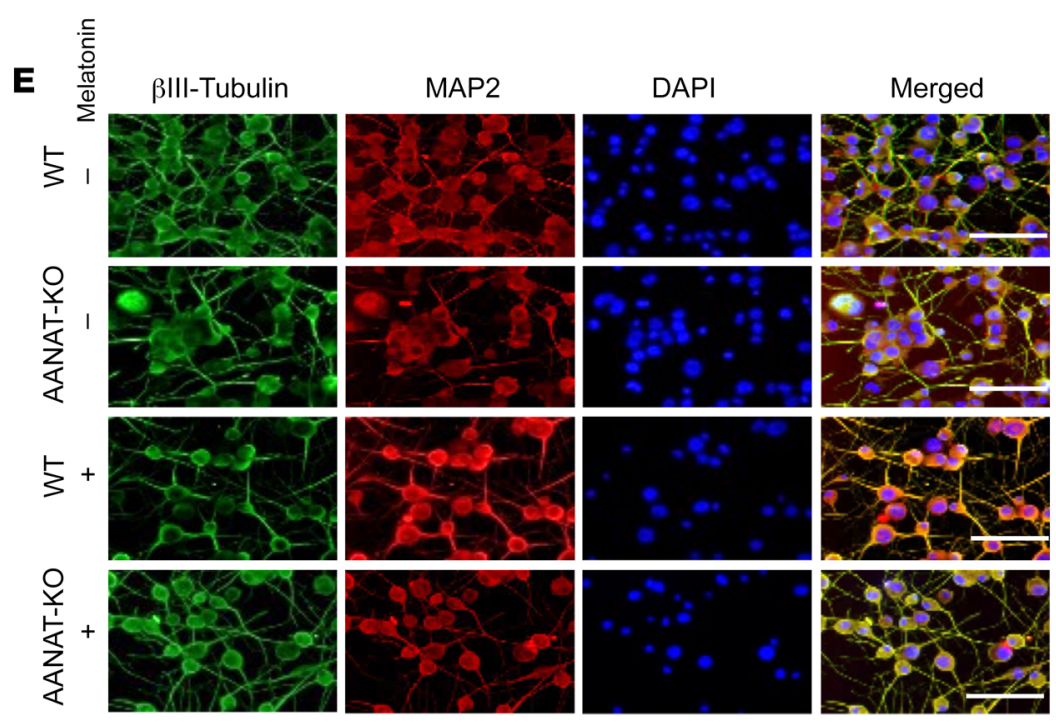

$\mathbf{F}$

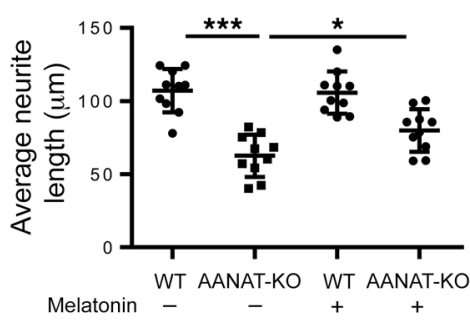

G

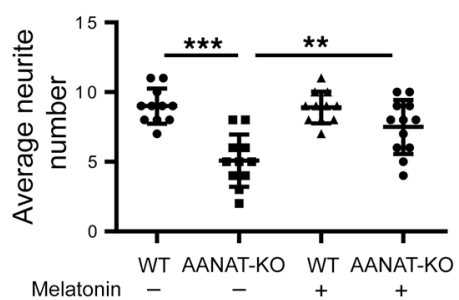


Figure 3. Melatonin inhibits neuroinflammation and degeneration in AANAT-KO-differentiated neurons. (A) qPCR analysis of cytosolic mtDNA in WT- and AANAT-KO-differentiated N2a cells grown with or without 5 $\mu \mathrm{M}$ melatonin using primers for $\mathrm{mt}-\mathrm{CO1}$, mt-Dloop1, and mt-Dloop3. Cytosolic mtDNA is plotted relative to the amount of WT N2a cells after normalization to $\beta$-actin from the corresponding total DNA lysate $(n=4)$. (B) Representative immunoblots and (C) quantification of cGAS, STING, IRF3, pNF- $\kappa B$, caspase-1, and $\beta$-actin in WT- and AANAT-KO-differentiated N2a cells grown with or without $5 \mu \mathrm{M}$ melatonin. $\beta$-actin was a loading control and protein levels are expressed relative to untreated WT $(n=3)$. (D) Cytokine ELISA in culture medium of WT- and AANAT-KO-differentiated N2a cells grown with or without $5 \mu \mathrm{M}$ melatonin $(n=3)$. For all experiments, WT and AANAT-KO N2a cells were differentiated by $10 \mu \mathrm{M}$ RA exposure for 8 days. (E) Representative images of immunocytochemical analysis of $\beta I I I-t u b u l i n$ in WT- and AANAT-KO-differentiated N2a cells grown with or without $5 \mu \mathrm{M}$ melatonin. Scale bars: $25 \mu \mathrm{m}$. (F) Average neurite length and (C) average neurite number analysis by neuron J logarithm using Image $J$ in $\beta$ III-tubulin-stained WT- and AANAT-KO-differentiated N2a cells grown with or without $5 \mu \mathrm{M}$ melatonin ( $n 3$, total 10 view fields). Data are expressed as mean \pm SD and analyzed by ANOVA followed by Tukey's test. ${ }^{*} P<0.05 ;{ }^{* *} P<0.01 ;{ }^{* * *} P<0.001$.

Depletion of mtDNA inhibits the hyperinflammatory response in AANAT-KO-differentiated neurons. To evaluate the role of cytosolic mtDNA in the inflammatory phenotype of N2a cells, we depleted their mtDNA. WT and AANAT-KO N2a cells were cultured with ethidium bromide (EtBr) to generate mtDNA-deficient N2a cells $(\rho 0)$. Chronic EtBr exposure results in reduction of mtDNA (56). We confirmed that EtBr exposure depleted mtDNA in N2a cells (Supplemental Figure $5, \mathrm{~A}-\mathrm{C})$. mtDNA release is decreased in $\rho 0$ AANATKO neurons (Figure 4A). Interestingly, mtDNA depletion in AANATKO neurons reduced proinflammatory cytokine secretion (Figure 4B). Moreover, mtDNA depletion inhibited caspase-1, caspase-3, and caspase- 9 activation and bid cleavage (Figure $4, C$ and D). These data point to cytosolic mtDNA in neurons as a key proinflammatory mediator. mtDNA depletion did not alter mitochondrial ROS or MMP, indicating that mtDNA release is downstream to mitochondrial ROS production, decreased in MMP, and upstream from inflammatory signaling (Supplemental Figure 5, D and E).

Given the impact of mtDNA reduction on activation of inflammatory pathways and the connection between inflammatory signaling and synaptic pruning, we evaluated the role of mtDNA on synaptic dynamics. mtDNA-depleted AANAT-KO neurons demonstrated synaptic preservation, measured by increased PSD95 and synaptophysin (protein and mRNA) as compared with untreated AANAT-KO neurons, similar to what we observed with exogenous melatonin (Figure 4, C and D, and Supplemental Figure $5 F$ ). These results link mtDNA release with inflammation and synaptic degeneration.

DNAse1 inhibits the inflammatory response in HD. To identify whether cytosolic mtDNA contributes to the inflammatory response in $\mathrm{HD}$, we evaluated STHdh $\mathrm{Q}^{\mathrm{C} / \mathrm{Q7}}(\mathrm{Q} 7)$ and $\mathrm{STHdh} \mathrm{C}^{\mathrm{111} / \mathrm{Q} 111}$ (Q111) cells for a mutant huntingtin-dependent (mHTT-dependent) inflammatory phenotype. Q7 and Q111 are immortalized striatal cells from mice that had a knocked-in, expanded CAG repeat in the huntingtin gene. These cells express mHTT with 111 glutamine repeats and are used as a cellular HD model (57). Q7 and Q111 cells differentiated to mature striatal neurons were evaluated for mtDNA-mediated inflammation. Q111 cells had a higher cytosolic mtDNA concentration compared with Q7 cells (Figure 5A). We transfected Q7- and Q111-differentiated neurons with DNAse1 protein to degrade cytosolic DNA. DNAase1 transfection did not alter the elevated ROS and reduced MMP detected in Q111 cells (Figure 5, B and C), complementing the EtBr experiments that demonstrated that mtDNA release is downstream of mitochondrial oxidative stress and MMP dysfunction. cGAS/STING/ IRF3 levels increased in differentiated Q111 neurons, an effect inhibited by DNAse1 (Figure 5D). In addition, proinflammatory cytokine secretion, which was increased in Q111 differentiated neurons, was inhibited by DNAse1 (Figure 5E and Supplemental Figure 6). Therefore, cytosolic mtDNA mediates mHTT-induced cGAS pathway upregulation/activation.

Inflammatory response in $H D$ is mediated by cGAS. To confirm that the inflammatory response is mediated by cGAS, we treated differentiated Q7 and Q111 neurons with RU.521 (cGAS pharmacological inhibitor) $(58,59)$. RU.521 did not affect cGAS level; however, it significantly reduced STING and IRF3 levels in differentiated Q7 and Q111 neurons (Figure 6, A and B). Moreover, RU.521 exposure also significantly inhibited INF- $\alpha$ and IFN- $\beta$ levels in differentiated Q7 and Q111 neurons (Figure 6C). These results further confirm that the inflammatory response induced by cytosolic mtDNA is mediated by cGAS.

Melatonin regulates mtDNA-mediated inflammation in $H D$. We followed the in vitro data by examining the relationship between mHTT and mtDNA signaling in vivo. To evaluate whether cytosolic mtDNA induces neuroinflammation in HD, we analyzed striatum (grade 2) from a patient with HD and found cGAS/STING/ IRF3 signaling activation (Figure 7A). In vivo, we evaluated the R6/2 mouse model of HD at 9 weeks of age (mid-symptomatic stage) and assessed mtDNA oxidative damage in cortex and striatum. WT brain lysates, which possess mtDNA and nuclear DNA, have low levels of 8-OHDG (DNA oxidation marker) (Figure 7B). 8-OHDG in brain DNA cortex and striatum demonstrated a 1.4and 1.6-fold increase, respectively, in R6/2 brain (Figure 7B). However, nonsynaptosomal mitochondria DNA demonstrated a 1.8- and 3.5-fold increase 8-OHDG concentrations in R6/2 cortex and striatum, respectively, compared with WT (Figure 7B). In synaptosomal mitochondria, the level of oxidized DNA is 3.7- and 3.9fold higher in R6/2 cortex and striatum, respectively, suggesting that synaptic mitochondria are more likely to have damaged DNA (Figure 7B). We then quantified cytosolic mtDNA and found incremental accumulation of cytosolic mtDNA associated with disease progression (Figure 7C).

We identified melatonin as a regulator of mtDNA release and subsequent neuroinflammation. We previously demonstrated that melatonin reduces mHTT-induced pathology in vivo and in cells. Melatonin extends survival and reduces brain atrophy and neuroinflammatory pathways in R6/2 mice (30). This effect is not limited to mHTT-mediated toxicity; melatonin is also protective in ALS (31). It is of interest that melatonin levels decrease in humans with HD, even before symptom onset (44). Since melatonin is protective in R6/2 mice and melatonin is deficient in humans with $\mathrm{HD}$, we evaluated whether exogenous melatonin reduces mtDNA release and consequent inflammation using 6-week-old R6/2 mice injected with melatonin daily for 3 weeks. In addition to inhibition of caspase-1 activation, which was previously demonstrated 
A
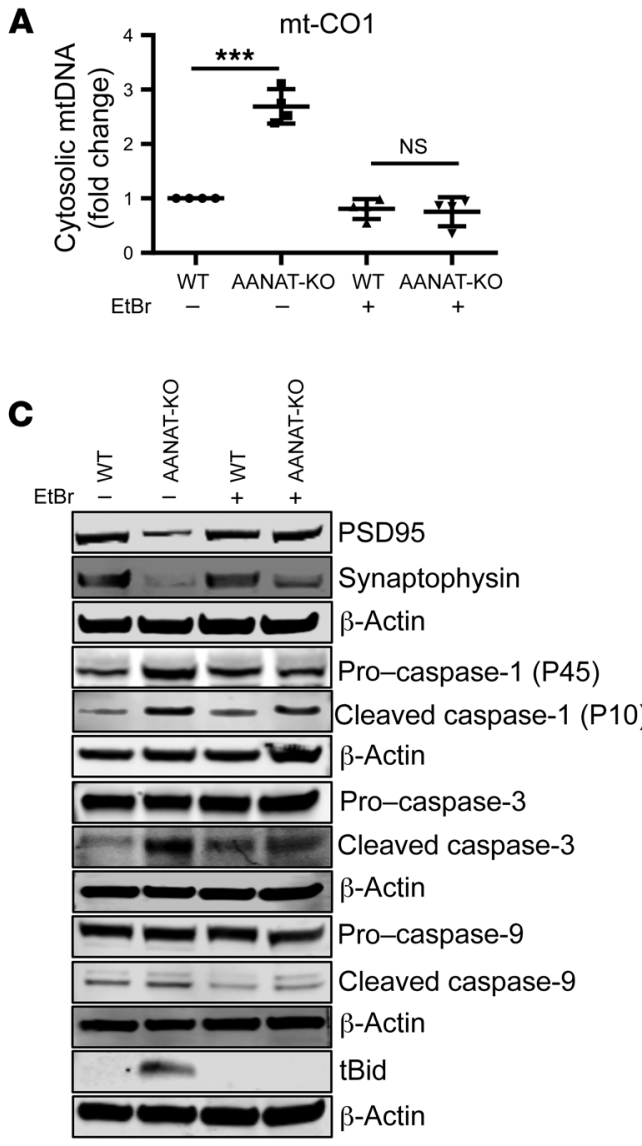

Figure 4. mtDNA depletion rescues synaptic degeneration and caspase activation in AANAT-KO-differentiated neurons. (A) qPCR of cytosolic mtDNA in WT- and AANAT-KOdifferentiated neurons grown with or without EtBr. Naive WT and AANAT-KO N2a cells were treated with $\mathrm{EtBr}(1 \mu \mathrm{g} /$ $\mathrm{mL}$ ) for 4 weeks to deplete mtDNA. Cytosolic mtDNA was measured using qPCR with primers for mt-C01, mt-Dloop1, and mt-Dloop3. Cytosolic mtDNA is plotted relative to the amount of untreated WT N2a after normalization to $\beta$-actin from the corresponding total DNA lysate $(n=4)$. (B) Cytokine ELISA in culture medium of WT- and AANAT-KOdifferentiated neurons and mtDNA depleted cells $(n=4)$. (C) Representative immunoblots and (D) quantification of PSD95, synaptophysin, caspase-1, caspase-3, caspase-9, tBid, and $\beta$-actin in WT- and AANAT-KO-differentiated neurons and their mtDNA-depleted form. $\beta$-actin was used as loading control $(n=3)$. For all experiments, untreated and mtDNA-depleted WT and AANAT-KO N2a cells were differentiated with $10 \mu \mathrm{M}$ RA exposure for 8 days. Data are expressed as mean \pm SD and analyzed by ANOVA followed by Tukey's test. ${ }^{*} P<0.05$; ${ }^{* *} P<0.01 ;{ }^{* *} P<0.001$.
B
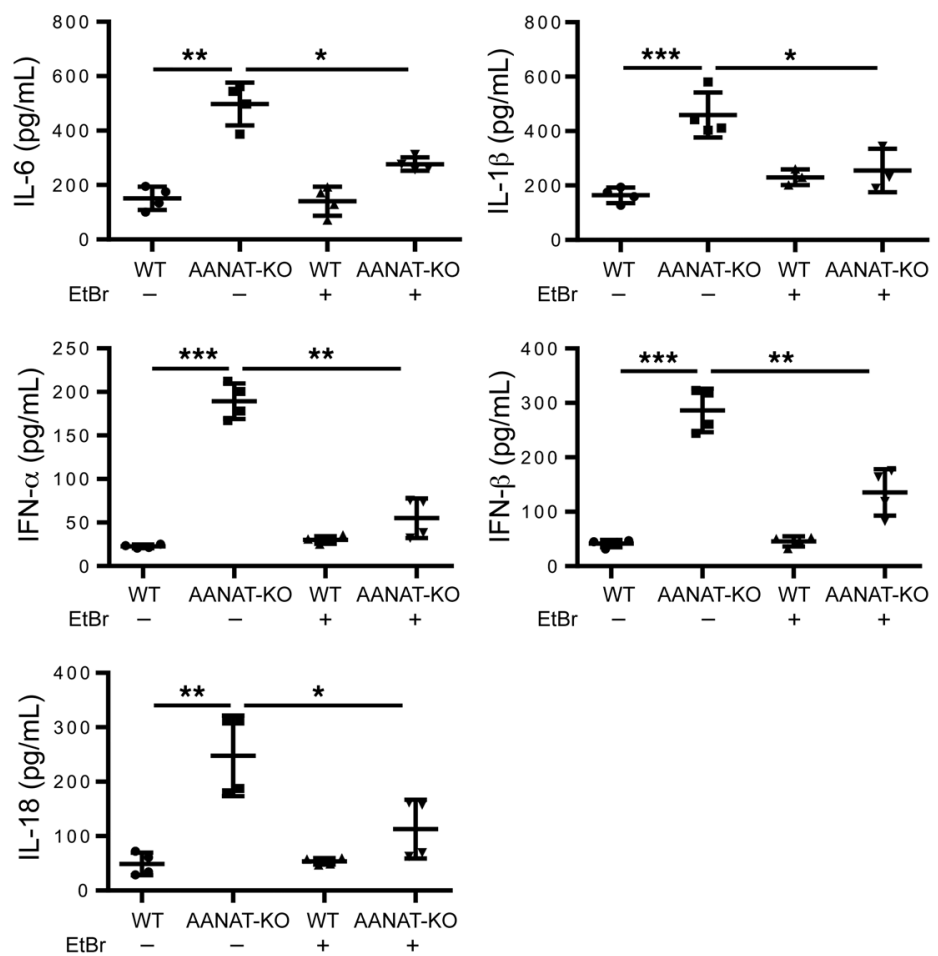

D
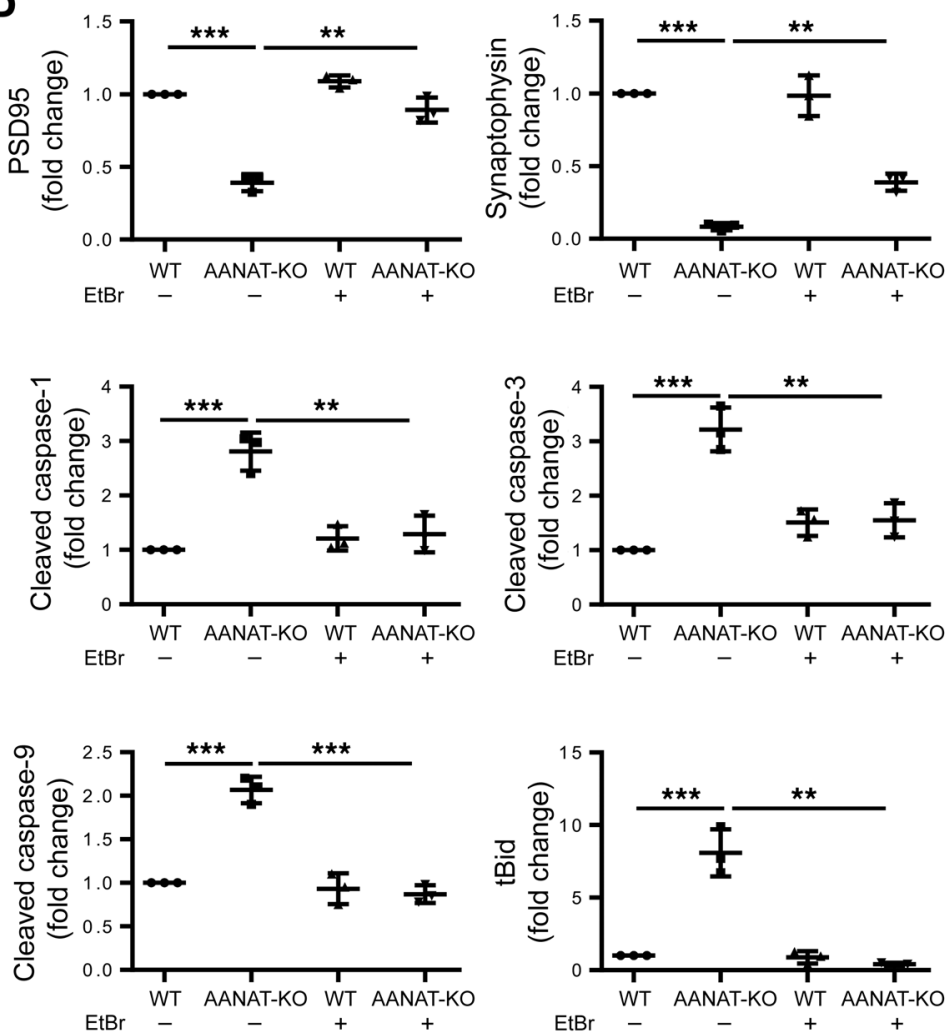

(30), melatonin inhibited mtDNA release and ameliorated cGAS/ STING/IRF3 upregulation in striatum of 9-week-old R6/2 mice (Figure 7, D and E, and Supplemental Figure 7A). Consistent with the inhibition of the master inflammatory regulators caspase-1 and cGAS, melatonin also reduced expression of proinflammatory cytokines in R6/2 brain (Figure 7F and Supplemental Figure 7B).

\section{Discussion}

Inflammation is a ubiquitous finding in neurodegenerative diseases; however, the proinflammatory trigger is not known. The cGAS intracellular DNA sensing pathway activates proinflammatory pathways in nonneural tissues (12-15). Using HD mice and mHTT-expressing cells, we believe we are first to demonstrate 
A

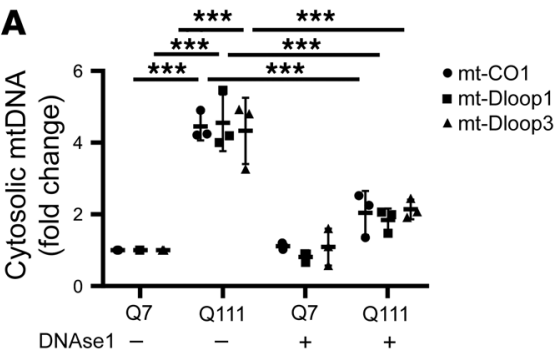

B

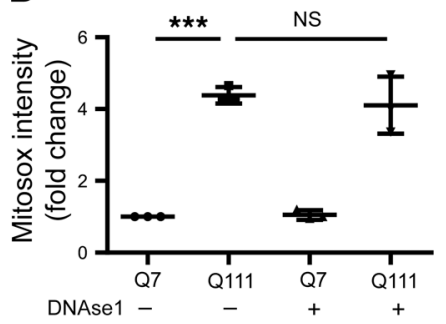

C

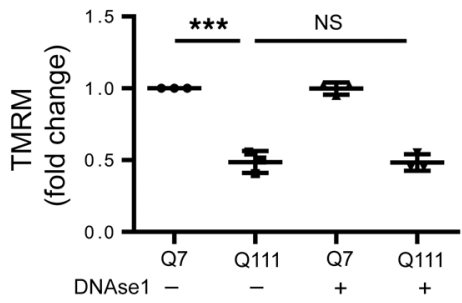

D
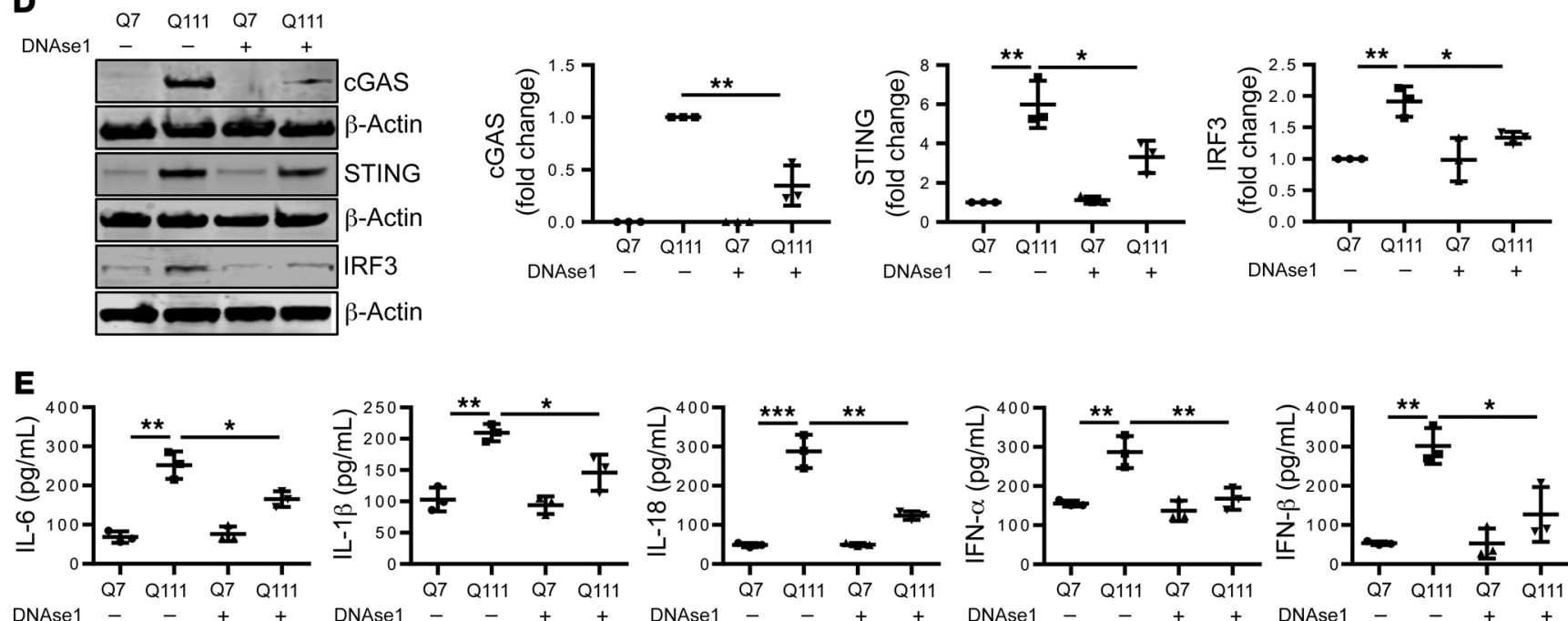

Figure 5. DNAse1 decreases expression of proinflammatory cytokine secretion in HD. (A) qPCR analysis of cytosolic mtDNA in differentiated Q7 and Q111 cells transfected with DNAse 1 or lactate dehydrogenase (LDH) as a control using mt-CO1, mt-Dloop1, and mt-Dloop3. Cytosolic mtDNA is plotted relative to the control transfected Q7-differentiated neurons after normalization to $\beta$-actin in the corresponding total DNA sample ( $n=3$ ). (B) Analysis of mitochondrial ROS by Mitosox in Q7- and Q111-differentiated neurons transfected with control LDH or DNAse 1. Mitosox fluorescence intensity is presented relative to fluorescence in Q7-differentiated neurons transfected with LDH $(n=3)$. (C) Analysis of MMP by TMRM in Q7- and Q111-differentiated neurons transfected with LDH control or DNAse $1(n=3)$. (D) Representative immunoblots and quantification of cGAS, IRF3, STING, and $\beta$-actin in Q7- and Q111-differentiated neurons transfected with LDH or DNAse $1(n=3)$. $\beta$-actin was used as endogenous normalization control. (E) Cytokine ELISA secretion in culture medium of differentiated Q7 and Q111 neurons transfected with LDH or DNAse1, shown as pg cytokine per mL culture medium ( $n=3$ ). Data are expressed as mean \pm SD and analyzed by ANOVA followed by Tukey's test. ${ }^{*} P<0.05 ;{ }^{* *} P<0.01 ;{ }^{* *} P<0.001$.

that cytosolic mtDNA in a neurological disease triggers cGAS signaling and subsequent production of proinflammatory cytokines. Complementary findings in early-stage human HD striatum demonstrates elevated levels of cGAS/STING/IRF3, providing human relevance and evidence that this is not late sequela of the degenerative process. Therefore, cytosolic mtDNA activates proinflammatory pathways in HD. Given that inflammation is a shared feature of neurodegenerative disease, it is likely that mtDNA release to the cytosol is a common mechanism among neurodegenerative diseases.

The fate of ROS-damaged mitochondria is either orderly disposal/recycling (mitophagy) or pathologic release of mtDNA and cell death mediators (cytochrome c, SMAC, AIF, EndoG). We demonstrate that melatonin deficits enhance mitochondrial ROS damage and subsequent mtDNA release with activation of cGAS leading to pathologic neuroinflammation. This finding is of particular relevance given that in neurons, melatonin is exclusively synthesized in mitochondrial matrix (35). Melatonin levels decrease with aging and in neurodegeneration, and melatonin supplementation is neuroprotective in experimental acute and chronic neuro- degenerative models (34, 60-62). Melatonin, at the pharmacologic dose used to exert antiinflammatory effects, likely saturates the melatonin receptor (63). Thus the melatonin effects likely include both receptor-mediated and non-receptor-mediated melatonin effects, including protecting mitochondria by scavenging ROS, inhibiting the mitochondrial permeability transition pore, and activating uncoupling proteins, which maintains the optimal mitochondrial membrane potential (37). Brain tissue from AANAT-KO mice have early free radical damage, increased caspase- 1 activation, and increased mtDNA release, resulting in cGAS-triggered neuroinflammation and premature neurodegeneration. These findings cement a key role for melatonin as a mediator of mitochondrial and neuronal health, and points to aging-associated melatonin loss in humans, which is exacerbated in neurodegeneration, as an important mechanism of neuronal vulnerability. Although the AANAT-KO mouse recapitulates features of accelerated aging (free radical damage, early mtDNA release, neuroinflammation, and neurodegeneration), establishing this mouse as a model of premature aging will require additional validation. Nonetheless, it recapitulates accelerated ROS-mediated dam- 
$\begin{array}{lllllll}\text { A } & \text { Q7 } & \text { Q111 } & \text { Q7 } & \text { Q111 } & \text { Q7 } & \text { Q111 }\end{array}$

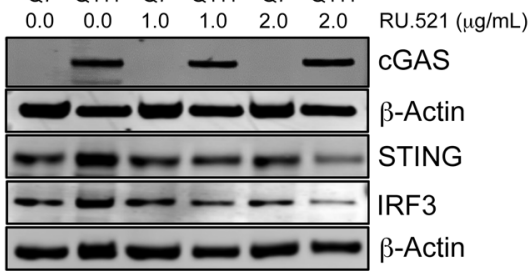

C

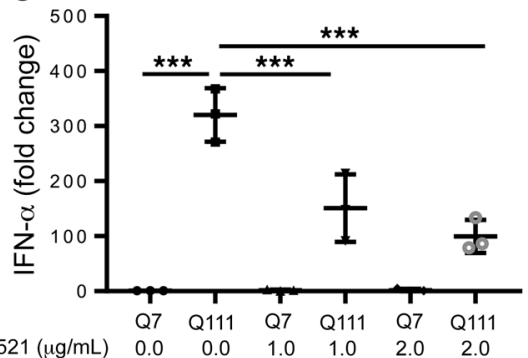

$\begin{array}{lllllll}\text { RU. } .521(\mu \mathrm{g} / \mathrm{mL}) & 0.0 & 0.0 & 1.0 & 1.0 & 2.0 & 2.0\end{array}$

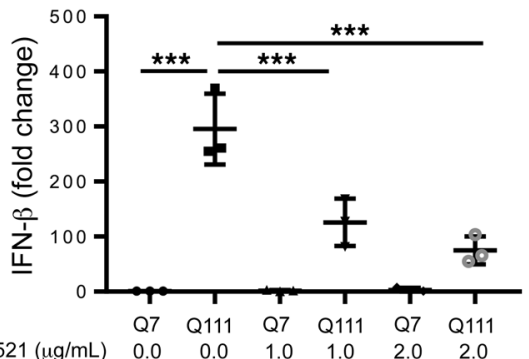

B
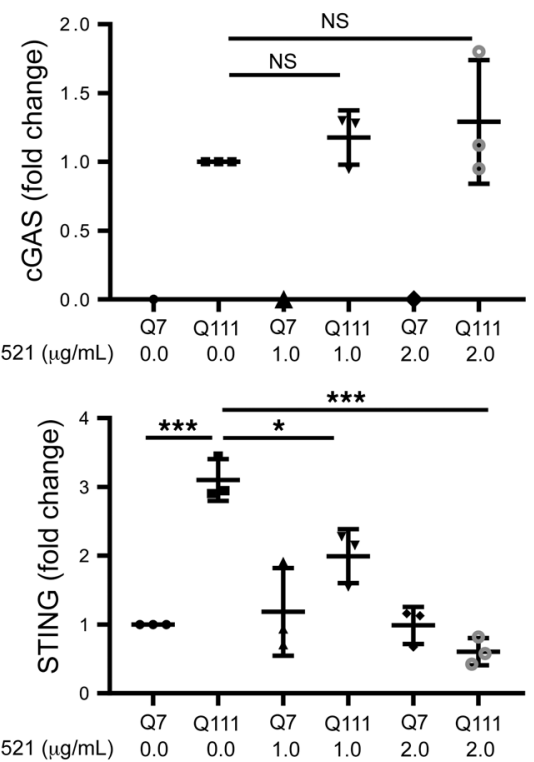

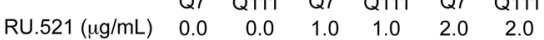

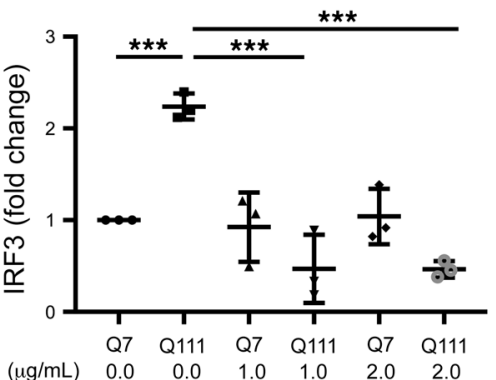

Figure 6. cGAS mediate mtDNA induced inflammation. (A) Representative immunoblots and (B) quantification of cGAS, IRF3, STING, and $\beta$-actin in Q7 and Q111 differentiated neurons exposed with RU.521 (cGAS inhibitor) $n=3, \beta$-actin was used as an endogenous normalization control. (C) qPCR analysis of IFN- $\alpha$ and IFN- $\beta$ mRNA in differentiated Q7 and Q111 cells treated with RU.521. The graph is plotted relative to the Q7-differentiated neurons after normalization to $\beta$-actin $(n=3)$. Data are expressed as mean \pm SD and analyzed by ANOVA. ${ }^{*} P<0.05$; ${ }^{* * *} P<0.001$. age in a WT background and is a tool for evaluation of the in vivo repercussions of this phenomenon.

In sum, we demonstrate that melatonin plays a key role in mitochondrial health, likely by blocking ROS damage and the resulting downstream consequences (i.e., mtDNA and cytochrome c release and caspase activation). Furthermore, cytosolic mtDNA plays a key role in activating inflammatory cytokine pathways, leading to synaptic pruning and neurodegeneration. These results provide a clear and direct link between mitochondrial damage, pathologic inflammation, synaptic degeneration, and neurodegeneration. We think that this is an evolving field. New agents to manipulate this pathway are being introduced and provide ongoing opportunities for in vitro and in vivo testing (64). Modulating these pathways may provide therapeutic options for aging and neurodegenerative diseases.

\section{Methods}

Human tissues. Striatum samples of grade 2 HD patients, and control patients' samples were obtained from the New York Brain Bank at Columbia University. Clinical information of the patients and subjects is indicated in Supplemental Table 1.

Murine tissues. All mouse tissues were collected in compliance with the NIH Guide for the Care and Use of Laboratory Animals. Animal protocols were approved by the University of Pittsburgh's Institutional Animal Care and Use Committee. All mice were kept in 12-hour light and dark cycle with ad libitum food and water. R6/2 mice, which carry the promoter sequence and exon 1 of a mutant human HTT gene with approximately $150 \mathrm{CAG}$ repeats, were obtained from the Jackson Laboratory. A colony was maintained by breeding R6/2 males with B6CBAF1 females (Jackson Laboratory). CAG repeat length was determined for every mouse in the colony. We used mice with $150 \pm 10 \mathrm{CAG}$ repeats. PCR genotyping was performed as previously described (65). The experiments used 3-, 6-, and 9-week-old R6/2 mice.

AANAT-KOmiceonCBAandDBAbackgroundweregeneratedusing CRISPR/Cas9 methods by the University of Pittsburgh Transgenic and Gene Targeting Laboratory and the Magee Women's Research Institute Genome Editing, Transgenic and Virus (GETV) Core Facility, respectively. CBA/J or DBA/2J embryos were injected with a mixture of Cas9 mRNA, AANAT-guide1-F sequence (TAATACGACTCACTATAGGTGATGTTCAACATGGGCGTCGTTTTAGAGCTAGAAATAGCA), and AANAT-guide2-F sequence (TAATACGACTCACTATAGGGGGAGACAgCGgtTCCCAacgttTtagagctagaAatagCA). From the injected zygotes, 2-cell embryos were transferred to the oviducts of pseudopregnant female recipients. Potential founder mice were genotyped and those with AANAT deletion were bred with WT mice (Jackson Laboratory) to determine germline transmission. Founders with germline transmission were bred with WT mice to generate heterozygotes. Heterozygotes were bred to generate littermate PCN and F2 KO and WT mice. Tissues were obtained from mice within our colony that were the age- and sex-matched offspring of $\mathrm{KO} \times \mathrm{KO}$ or WT $\times$ WT F3 breeding. A total of $190 \mathrm{CBA} / \mathrm{J}$ fertilized embryos were injected with a mixture of Cas 9 mRNA (100 ng/ $\mu \mathrm{L})$, AANAT-guide1-F (50 ng/ $\mu \mathrm{L})$, and AANAT-guide2-F (50 ng/ $\mu \mathrm{L})$. From the injected zygotes, 120 embryos developed to the 2-cell stage and were transferred to the oviducts of 5 
A
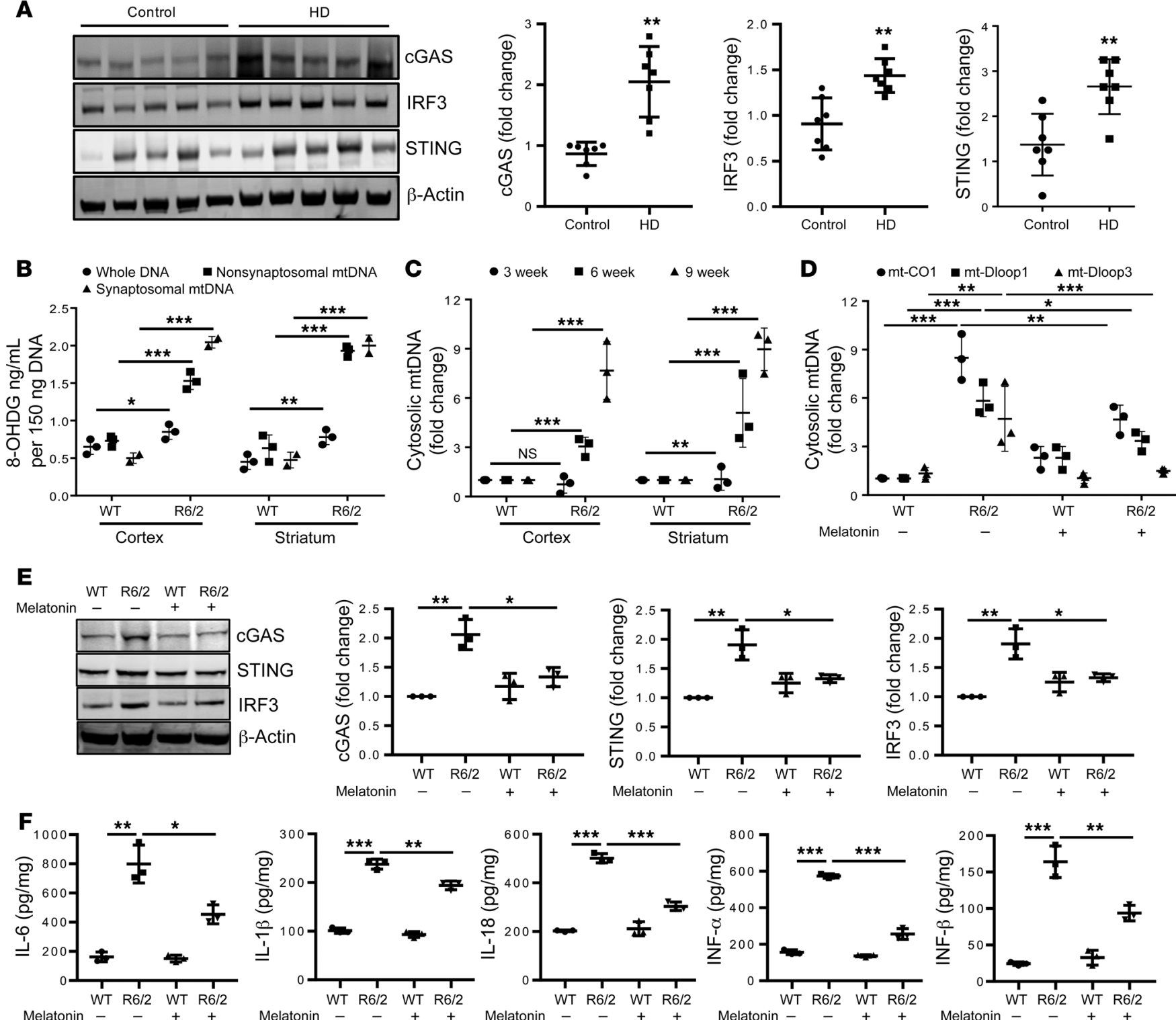

Figure 7. Melatonin inhibits mtDNA release and inflammation in HD. (A) Representative immunoblots and quantification of cGAS, IRF3, STING, and $\beta$-actin in human postmortem brain lysate of control and HD grade 2 striatum. Expression was normalized to $\beta$-actin and shown relative to control ( $n=7)$. (B) ELISA quantification of 8-OHDG, a marker of DNA oxidation, in total DNA, nonsynaptosomal mtDNA, and synaptosomal mtDNA in 9-week-old WT and R6/2 cortical and striatal brain tissue $(n=3)$. (C) qPCR analysis of cytosolic mtDNA in presymptomatic ( 3 weeks), early symptomatic ( 6 weeks), and mid-symptomatic (9 weeks) WT and R6/2 cortex and striatum. Cytosolic mtDNA is plotted relative to amount of WT cortex and striatum after normalization to $\beta$-actin from the corresponding total DNA sample $(n=3)$. (D) qPCR analysis of cytosolic mtDNA in WT and R6/2 striatum injected with melatonin $(30 \mathrm{mg} / \mathrm{kg}$ ) or vehicle from 6 to 9 weeks of age. Cytosolic mitochondrial genes are plotted relative to WT striatum after normalization to $\beta$-actin from the corresponding total DNA sample $(n=3)$. (E) Representative immunoblots and quantification of cGAS, IRF3, STING, and $\beta$-actin in 9-week-old WT and R6/2 striatum injected with melatonin or vehicle. Expression was normalized to $\beta$-actin and shown relative to vehicle treated control ( $n=3)$. (F) Cytokine ELISA in WT and R6/2 cortex injected with melatonin or vehicle. Data shown as pg cytokine per mg of protein lysate $(n=3)$. Data are expressed as mean \pm SD and analyzed by Student's $t$ test $(\mathbf{A})$ or ANOVA followed by Tukey's test $(\mathbf{B}-\mathbf{F}){ }^{*} P<0.05 ;{ }^{* *} P<0.01 ;{ }^{* *} P<0.001$.

pseudopregnant female recipients. The pseudopregnant female recipients gave birth to 19 pups. Genotyping identified 5 possible founders. These were bred with WT CBA/J, and 2 confirmed germline transmission. These 2 founder lines were maintained separately.

Cell culture and primary neuronal cultures. N2a cells were obtained from ATCC and grown in DMEM/F12 medium with 10\% FBS as described in earlier studies (66). AANAT-KO N2a cells were generated as described in our previous study (35). Q7 and Q111 knockin mouse striatal cell lines that express full-length WT (polyQ7) and mutant (polyQ111) HTT were a gift from Marci MacDonald (Harvard Medical School, Boston, Massachusetts, USA), who generated these lines (57). Q7 and Q111 cells were grown in MEM culture medium with $10 \%$ FBS. AANAT-KO and littermate WT PCN cultures were prepared from E15.5 embryos, as previously described (27).

Cell differentiation. N2a cells were differentiated by exposing them to $10 \mu \mathrm{M}$ retinoic acid (RA) with $2 \%$ fetal bovine serum (FBS) 
for 8 days. Every alternate day, the culture medium of differentiating $\mathrm{N} 2 \mathrm{a}$ cells was replaced with fresh medium containing $10 \mu \mathrm{M}$ RA. N2a cells were treated with $5 \mu \mathrm{M}$ melatonin during differentiation to evaluate the effect of melatonin in WT- and AANAT-KO-differentiated N2a neurons. Fresh melatonin was added with RA every alternate day for 8 days. All experiments were performed in 8-day-differentiated neurons with or without melatonin unless otherwise specified. Differentiation of Q7 and Q111 cells was induced by exposing cells to 10 $\mathrm{ng} / \mathrm{mL} \alpha$-FGF, $250 \mu \mathrm{M}$ 3-isobutyl-1-methylxanthine (IBMX), $200 \mathrm{nM}$ phorbol 12-myristate 13-acetate (TPA), $50 \mu \mathrm{M}$ forskolin, and $10 \mu \mathrm{M}$ dopamine for 2 days.

cGAS inhibitor treatment. RU.521 (Invivogen) was dissolved in DMSO as $2 \mathrm{mg} / \mathrm{mL}$ stock solution. Q7 and Q111 cells were treated with RU.521 either by $1 \mathrm{mg} / \mathrm{mL}$ or $2 \mathrm{mg} / \mathrm{mL}$ RU.521 or DMSO in a control group as vehicle before 24 hours of differentiation. After 24 hours of cell culture, media was replaced with differentiation media (as described above) having RU.521. After differentiation, cells were collected for protein and mRNA analysis.

Brain and ventricle size analysis. Histological staining and stereotactic analysis were done as previously described (67). AANAT-KO and littermate control mice were deeply anesthetized and transcardially perfused with $4 \%$ buffered paraformaldehyde. The brains were removed and postfixed in the perfusate overnight, rinsed in buffer, weighed, and cryoprotected in a graded series of $10 \%$ and $20 \%$ glycerol/2\% DMSO. Frozen serial sections were cut at $50 \mathrm{~mm}$, stored in 6-well plates, and stained for Nissl substance by using cresyl violet. Serial-cut coronal tissue sections (every fourth section) from the rostral segment of the neostriatum to the level of the anterior commissure (interaural $5.34 \mathrm{~mm} /$ bregma $1.54 \mathrm{~mm}$ to interaural $3.7 \mathrm{~mm} /$ bregma $0.10 \mathrm{~mm}$ ) were used for volumetric analysis. Images were captured by a Nikon E800 microscope with SPOT Flex camera and analyzed with NIH ImageJ software.

Melatonin detection. Mouse pineal gland, differentiated N2a cell pellet, and isolated mitochondria were resuspended in $500 \mu \mathrm{L}$ of 1xPBS solution with protease inhibitor set III (Millipore, 539134) and MAO-A inhibitor (clorgyline, M3778, Sigma-Aldrich), followed by sonication on ice (3 strokes each). All samples were analyzed using ultra performance liquid chromatography-tandem mass spectometry (UPLC-MS/MS). The UPLC-MS/MS method for quantitation of melatonin employs liquid-liquid extraction and detection with a triple quad mass spectrometer. To each $0.5-\mathrm{mL}$ sample, we added $50 \mu \mathrm{L}$ ammonium hydroxide and $10 \mu \mathrm{L}$ internal standard $\left(\mathrm{d}_{4}\right.$ melatonin $\left.50 \mathrm{ng} / \mathrm{mL}\right)$. Samples were vortexed and extracted with $2 \mathrm{~mL}$ methylene chloride. After centrifugation, the methylene chloride was evaporated under a gentle stream of nitrogen and the residue reconstituted in $50 \mu \mathrm{L} \mathrm{H}_{2} \mathrm{O}$ for injection onto an Acquity UPLC BEH C18 column $(2.1 \times 100 \mathrm{~mm})$. Samples were eluted with $2 \mathrm{mM}$ ammonium formate in water and acetonitrile using a gradient from 97:3 (initial water/ACN) to 60:40 over 5 minutes and maintained at 60:40 for 0.5 minutes before reequilibration at 97:3. Total run time was 7.5 minutes. Melatonin and the internal standard were detected in the positive mode with a Thermo Fisher TSQ Quantum Ultra mass spectrometer interfaced via an electrospray ionization probe with the Waters UPLC Acquity solvent delivery system. Transitions used for analysis were $237.1 \rightarrow 178.1$ for $\mathrm{d}_{4}$ melatonin and $233.1 \rightarrow 174.1$ for melatonin. The method may be used to quantify melatonin in brain homogenates, cell culture media, cell lysates, or serum/plasma samples. Calibrators are extracted from PBS buffer, cell culture media, or stripped human plasma to match the sample matrix. The lower limit of quantitation is $1 \mathrm{pg} / \mathrm{mL}$. The method was adapted from Wang et al. (68).

Generation of $m t D N A$-deficient cells. To generate the mtDNA-deficient phenotype, N2a cells were grown in DMEM/F12 supplemented with $10 \%$ (vol/vol) FBS, $100 \mu \mathrm{g} / \mathrm{mL}$ pyruvate, $50 \mu \mathrm{g} / \mathrm{mL}$ uridine, and $1 \mu \mathrm{g} / \mathrm{mL}$ EtBr for 3 weeks. mtDNA depletion was evaluated by quantitative real-time PCR and immunoblots of mtDNA-encoded proteins.

Immunostaining. Cells were fixed in $4 \%$ paraformaldehyde in PBS for 10 minutes, permeabilized with $0.1 \%$ Triton X-100 in PBS for 15 minutes at room temperature, and subjected to immunofluorescence with the indicated primary and secondary antibodies. Samples were imaged with the Olympus IX-81-DSU in confocal mode.

ROS, MMP, and MPT assays. ROS accumulation in cells was estimated by either DCFDA ( $2^{\prime}, 7^{\prime}$-dichlorofluorescin diacetate) for total ROS or Mitosox for mitochondrial ROS. Cells were incubated in $20 \mu \mathrm{M}$ DCFDA for 45 minutes or $5 \mu \mathrm{M}$ Mitosox for 15 minutes at $37^{\circ} \mathrm{C}$, and fluorescence was measured using plate reader or flow cytometry. For MMP estimation, JC-1 (tetraethylbenzimidazolylcarbocyanine iodide) or TMRM (tetramethylrhodamine methyl ester) was used. Cells were incubated in $2 \mu \mathrm{g} / \mathrm{mL} \mathrm{JC}-1$ or $25 \mathrm{nM}$ TMRM for 30 minutes at $37^{\circ} \mathrm{C}$, and fluorescence was measured using plate reader or flow cytometry. MPT was assayed using the MitoProbe Transition Pore Assay Kit (Invitrogen) according to the manufacturer's protocol.

Mitochondrial isolation. Brain mitochondria were isolated by Percoll density centrifugation as described in our previous studies $(27,53)$. Cellular mitochondria were isolated by using the mouse Mitochondria Isolation kit (Miltenyi Biotec) according to the manufacturer's protocol. In brief, $1 \times 10^{7}$ cells were collected and centrifuged at $300 \mathrm{~g}$ for 5 minutes and washed with PBS. After washing, cells were lysed in $1 \mathrm{~mL}$ ice-cold lysis buffer by passing through a 27 -gauge needle 15 times on ice. After lysis, lysate was incubated with $50 \mu \mathrm{L}$ anti-Tom 22 microbeads in $1 \times$ separation buffer for 1 hour at $4^{\circ} \mathrm{C}$. Suspension was then passed through a preseparation filter (Miltenyi Biotec) on LS column and washed with $1 \times$ separation buffer. Mitochondria were eluted with IM-2 (Isolation Buffer 2, $225 \mathrm{mM}$ sucrose, $75 \mathrm{mM}$ mannitol, $5 \mathrm{mM}$ HEPES, pH 7.4 at $4^{\circ} \mathrm{C}$ ) and centrifuged at $13,000 \mathrm{~g}$ for 4 minutes at $4^{\circ} \mathrm{C}$.

Immunoblotting. Samples (isolated mitochondria or whole-cell lysates) were cleared by centrifugation at 20,000 $\mathrm{g}$, equal amounts of protein were separated on Novex $4 \%-12 \%$ gradient polyacrylamide gels (Invitrogen), and proteins were transferred overnight onto 0.45$\mu \mathrm{m}$ polyvinylidene difluoride (PVDF) membranes. The membranes were then incubated with the indicated primary antibodies overnight at $4^{\circ} \mathrm{C}$ followed by incubation with secondary antibodies (Li-Cor) for 1 hour at room temperature, which were detected using the Odyssey CLx Infrared Imaging System (Li-Cor). Band intensities were quantified using Image Studio (Li-Cor). All the details for antibody used in immunoblotting are in Supplemental Table 2.

RNA isolation and quantitative PCR. Total RNA was isolated using the RNeasy Plus kit (Qiagen) and quantified using an ND-1000 spectrophotometer. cDNA was prepared using 1000 ng total RNA by reverse transcription PCR (RT-PCR) using a high-capacity cDNA reverse transcription kit (Applied Biosystems) according to the manufacturer's instructions. Quantitative PCR (qPCR) was performed on cDNA using SYBR Green chemistry. qPCR was performed on a Biorad CFX touch PCR using Applied Biosystems PowerUp SYBR Green master mix. For SYBR primer pair sequences, see Supplemental Table 1. 
Fold changes in expression were calculated by the $\Delta \Delta \mathrm{Ct}$ method using mouse $\beta$-actin as an endogenous control for mRNA expression. All fold changes were expressed normalized to the untreated control. Primers used are listed in Supplemental Table 3.

Cytosolic mtDNA quantification. For measurement of mtDNA in cytosol in cells, cell fractions were prepared using the Cell Fractionation Kit (Abcam) according to the manufacturer's protocol. For measurement of cytosolic mtDNA in brain, tissues were homogenized in IM buffer (5 mM HEPES-Tris [pH 7.4], $225 \mathrm{mM}$ sucrose, $75 \mathrm{mM}$ mannitol, and $1 \mathrm{mM}$ EGTA) and divided into 2 equal parts. One part is then centrifuged at $1300 \mathrm{~g}$ for 3 minutes and supernatant again spun at 20,000 $\mathrm{g}$ for $10 \mathrm{~min}$ utes. Resultant supernatant was collected as cytosolic fraction. Cytosolic fraction $(200 \mu \mathrm{L})$ and corresponding total tissue or cellular homogenate were used to isolate cytosolic and total DNA, respectively. The copy number of mtDNA-encoding cytochrome c oxidase 1 (mt-CO1), mt-Dloop1, and mt-Dloop3 was measured by qPCR with the same volume of DNA solution. Normalization to the nuclear genome was performed using DNA isolated from tissue homogenate using $\beta$-actin.

Mitochondrial in vitro protein import assay. The assay was performed as previously described (20). Ornithine transcarbamylase (OTC) precursor cDNA in pGEM-3Zf ${ }^{+}$pOTC plasmid was transcribed and translated in vitro using the TNT Coupled Reticulocyte Lysate System (Promega) in the presence of $\left[{ }^{35} \mathrm{~S}\right]$ methionine (PerkinElmer). Following translation, $\left[{ }^{35} \mathrm{~S}\right]$ methionine-labeled pOTC was incubated with isolated mitochondria at $25^{\circ} \mathrm{C}$ for the indicated times, and mitochondria containing imported OTC were collected by centrifugation (9000g, 10 minutes) and subjected to SDS-PAGE. Mature OTC, which represents the cleaved protein after translocation into the mitochondrial matrix, was quantified by Image $(\mathrm{NIH})$. The data are presented as normalized by input (total $\left[{ }^{35} \mathrm{~S}\right]$ pOTC per lane).

Protein transfection. Q7 and Q111 cells were plated in 6-well plates a day before protein transfections. Cells were washed twice with warm DMEM without FBS, then transfected for 4 hours at $37^{\circ} \mathrm{C}$ with $2 \mu \mathrm{g} /$ $\mathrm{mL}$ DNAse I or lactate dehydrogenase as a nontargeted protein control through the use of PULSin reagent (Polyplus Transfection). After 4 hours, transfection medium was removed and cells were incubated for 2 hours in complete growth media, and differentiation was induced by differentiation media as described above.

Statistics. Statistical analyses were performed with GraphPad Prism 7.0. Data were obtained from at least 3 independent experi- ments and expressed as mean \pm SD unless otherwise specified. The 2-tailed Student's $t$ test for parametric data and paired $t$ tests were used for experiments with multiple samples from the same source. One-way ANOVA or 2-way ANOVA followed by Tukey's test were used for analysis of more than 2 groups as specified in each figure legends. $P$ values less than 0.05 were considered statistically significant $\left({ }^{*} P<0.05 ;{ }^{* *} P<0.01 ;{ }^{* * *} P<0.001\right)$.

Study approval. All mouse work was approved by the University of Pittsburgh Institutional Animal Care and Use Committee (protocol 18011898). Cortex and striatal samples from patients with HD and control patients were obtained from the New York Brain Bank at Columbia University after approval from the University of Pittsburgh Committee for Oversight of Research Involving Decedents (CORID).

\section{Author contributions}

AJ, SVB, JK, MBM, SMP, DLC, and RMF designed experiments. AJ, SVB, YS, TS, SY, JK, FL, XW, and PO generated data. AJ, DLC, and RMF wrote the manuscript. RMF conceived the initial study.

\section{Acknowledgments}

We acknowledge Chunming Bi and Sebastien Gringas at the University of Pittsburgh Transgenic and Gene Targeting Laboratory for generating the CBA/J AANAT-KO founder mice and Yi Sheng and Kyle Orwig of the MWRI Genome Editing, Transgenic and Virus (GETV) Core Facility for generating the DBA/J2 AANATKO mice. We thank Jean Paul Vonsattel at Columbia University for his assistance in providing brain samples. This project used the HPLC-MS services of the University of Pittsburgh Small Molecule Biomarker Core and related sample analysis was supported by NIH S10RR023461-01 NCRRAPI4000 QTRAP (to SP). We acknowledge funding from NIH grant R01NS100743 (to RMF), the Clear Thoughts Foundation (to RMF), and the Pittsburgh Foundation Walter L. Copeland Fund UN2018-96996 (to AJ). Human brain tissues were obtained with the support of NIH grant P50AG008702 to the New York Brain Bank at Columbia University/Taub Institute.

Address correspondence to: Robert M. Friedlander, 200 Lothrop Street, B400 Presbyterian Hospital, Pittsburgh, Pennsylvania 15213, USA. Phone: 412.647.6358; Email: friedlanderr@upmc.edu.
1. Rolig RL, McKinnon PJ. Linking DNA damage and neurodegeneration. Trends Neurosci. 2000;23(9):417-424.

2. Yang JL, Weissman L, Bohr VA, Mattson MP. Mitochondrial DNA damage and repair in neurodegenerative disorders. DNA Repair (Amst). 2008;7(7):1110-1120.

3. Madabhushi R, Pan L, Tsai LH. DNA damage and its links to neurodegeneration. Neuron. 2014;83(2):266-282.

4. Acevedo-Torres K, et al. Mitochondrial DNA damage is a hallmark of chemically induced and the R6/2 transgenic model of Huntington's disease. DNA Repair (Amst). 2009;8(1):126-136.

5. Lin MT, Beal MF. Mitochondrial dysfunction and oxidative stress in neurodegenerative diseases. Nature. 2006;443(7113):787-795.

6. Yakes FM, Van Houten B. Mitochondrial DNA damage is more extensive and persists longer than nuclear DNA damage in human cells following oxidative stress. Proc Natl Acad Sci USA 1997;94(2):514-519.

7. Mecocci P, MacGarvey U, Beal MF. Oxidative damage to mitochondrial DNA is increased in Alzheimer's disease. Ann Neurol. 1994;36(5):747-751.

8. Nakahira K, et al. Autophagy proteins regulate innate immune responses by inhibiting the release of mitochondrial DNA mediated by the NALP3 inflammasome. Nat Immunol. 2011;12(3):222-230.

9. West AP, Shadel GS. Mitochondrial DNA in innate immune responses and inflammatory pathology. Nat Rev Immunol. 2017;17(6):363-375.

10. Gao D, et al. Cyclic GMP-AMP synthase is an innate immune sensor of HIV and other retroviruses. Science. 2013;341(6148):903-906.
11. Sun L, Wu J, Du F, Chen X, Chen ZJ. Cyclic GMPAMP synthase is a cytosolic DNA sensor that activates the type I interferon pathway. Science. 2013;339(6121):786-791.

12. Chen Q, Sun L, Chen ZJ. Regulation and function of the cGAS-STING pathway of cytosolic DNA sensing. Nat Immunol. 2016;17(10):1142-1149.

13. Cai X, Chiu YH, Chen ZJ. The cGAS-cGAMPSTING pathway of cytosolic DNA sensing and signaling. Mol Cell. 2014;54(2):289-296.

14. Xiao TS, Fitzgerald KA. The cGAS-STING pathway for DNA sensing. Mol Cell. 2013;51(2):135-139.

15. Li T, Chen ZJ. The cGAS-cGAMP-STING pathway connects DNA damage to inflammation, senescence, and cancer. J Exp Med. 2018;215(5):1287-1299.

16. van de Veerdonk FL, Netea MG, Dinarello CA, Joosten LA. Inflammasome activation and IL-1 $\beta$ 
and IL-18 processing during infection. Trends Immunol. 2011;32(3):110-116.

17. Wishart TM, Parson SH, Gillingwater TH. Synaptic vulnerability in neurodegenerative disease. J Neuropathol Exp Neurol. 2006;65(8):733-739.

18. Perry VH, Nicoll JA, Holmes C. Microglia in neurodegenerative disease. Nat Rev Neurol. 2010;6(4):193-201.

19. Friedlander RM, Brown RH, Gagliardini V, Wang J, Yuan J. Inhibition of ICE slows ALS in mice. Nature. 1997;388(6637):31.

20. Friedlander RM, et al. Expression of a dominant negative mutant of interleukin- 1 beta converting enzyme in transgenic mice prevents neuronal cell death induced by trophic factor withdrawal and ischemic brain injury. J Exp Med. 1997;185(5):933-940.

21. Ona VO, et al. Inhibition of caspase-1 slows disease progression in a mouse model of Huntington's disease. Nature. 1999;399(6733):263-267.

22. Li M, et al. Functional role of caspase-1 and caspase-3 in an ALS transgenic mouse model. Science. 2000;288(5464):335-339.

23. Fink KB, et al. Reduction of post-traumatic brain injury and free radical production by inhibition of the caspase-1 cascade. Neuroscience. 1999;94(4):1213-1218.

24. Li M, et al. Functional role and therapeutic implications of neuronal caspase- 1 and -3 in a mouse model of traumatic spinal cord injury. Neuroscience. 2000;99(2):333-342.

25. Zhang WH, et al. Fundamental role of the Rip2/ caspase-1 pathway in hypoxia and ischemiainduced neuronal cell death. Proc Natl Acad Sci USA. 2003;100(26):16012-16017.

26. [no authors listed]. A novel gene containing a trinucleotide repeat that is expanded and unstable on Huntington's disease chromosomes. The Huntington's Disease Collaborative Research Group. Cell.1993;72(6):971-983.

27. Yano H, et al. Inhibition of mitochondrial protein import by mutant huntingtin. Nat Neurosci. 2014;17(6):822-831.

28. Yablonska S, et al. Mutant huntingtin disrupts mitochondrial proteostasis by interacting with TIM23. Proc Natl Acad Sci USA. 2019;116(33):16593-16602.

29. Wang X, et al. Methazolamide and melatonin inhibit mitochondrial cytochrome $\mathrm{C}$ release and are neuroprotective in experimental models of ischemic injury. Stroke. 2009;40(5):1877-1885.

30. Wang X, et al. The melatonin MT1 receptor axis modulates mutant Huntingtin-mediated toxicity. J Neurosci. 2011;31(41):14496-14507.

31. Zhang Y, et al. Melatonin inhibits the caspase-1/ cytochrome c/caspase-3 cell death pathway, inhibits MT1 receptor loss and delays disease progression in a mouse model of amyotrophic lateral sclerosis. Neurobiol Dis. 2013;55:26-35.

32. Reiter RJ, Sainz RM, Lopez-Burillo S, Mayo JC, Manchester LC, Tan DX. Melatonin ameliorates neurologic damage and neurophysiologic deficits in experimental models of stroke. Ann N Y Acad Sci. 2003;993:35-47.

33. Wakatsuki A, Okatani Y, Izumiya C, Ikenoue N. Melatonin protects against ischemia and reperfusion-induced oxidative lipid and DNA damage in fetal rat brain. J Pineal Res. 1999;26(3):147-152.

34. Wang $X$, et al. The melatonin MT1 receptor axis modulates mutant Huntingtin-mediated toxicity. J Neurosci. 2011;31(41):14496-14507.

35. Suofu Y, et al. Dual role of mitochondria in producing melatonin and driving GPCR signaling to block cytochrome c release. Proc Natl Acad Sci USA. 2017;114(38):E7997-E8006.

36. Zhang HM, Zhang Y. Melatonin: a well-documented antioxidant with conditional pro-oxidant actions. J Pineal Res. 2014;57(2):131-146.

37. Tan DX, Manchester LC, Qin L, Reiter RJ. Melatonin: A mitochondrial targeting molecule involving mitochondrial protection and dynamics. Int J Mol Sci. 2016;17(12):E2124.

38. Reiter RJ, Tan DX, Manchester LC, El-Sawi MR. Melatonin reduces oxidant damage and promotes mitochondrial respiration: implications for aging. Ann N Y Acad Sci. 2002;959:238-250.

39. Acuña-Castroviejo D, et al. Melatonin, mitochondria, and cellular bioenergetics. JPineal Res. 2001;30(2):65-74.

40. Wang $X$, et al. Inhibitors of cytochrome c release with therapeutic potential for Huntington's disease. J Neurosci. 2008;28(38):9473-9485.

41. Lee EJ, et al. Melatonin attenuates gray and white matter damage in a mouse model of transient focal cerebral ischemia. JPineal Res. 2005;38(1):42-52.

42. Tyagi E, Agrawal R, Nath C, Shukla R. Effect of melatonin on neuroinflammation and acetylcholinesterase activity induced by LPS in rat brain. Eur J Pharmacol. 2010;640(1-3):206-210.

43. Esposito E, Cuzzocrea S. Antiinflammatory activity of melatonin in central nervous system. Curr Neuropharmacol. 2010;8(3):228-242.

44. Kalliolia E, et al. Plasma melatonin is reduced in Huntington's disease. Mov Disord. 2014;29(12):1511-1515.

45. Liu RY, Zhou JN, van Heerikhuize J, Hofman MA, Swaab DF. Decreased melatonin levels in postmortem cerebrospinal fluid in relation to aging, Alzheimer's disease, and apolipoprotein E-epsilon4/4 genotype. JClin Endocrinol Metab. 1999;84(1):323-327.

46. Goto M, Oshima I, Tomita T, Ebihara S. Melatonin content of the pineal gland in different mouse strains. J Pineal Res. 1989;7(2):195-204.

47. Kasahara T, Abe K, Mekada K, Yoshiki A, Kato T. Genetic variation of melatonin productivity in laboratory mice under domestication. Proc Natl Acad Sci USA. 2010;107(14):6412-6417.

48. Klein DC. Arylalkylamine N-acetyltransferase: "the Timezyme”. J Biol Chem. 2007;282(7):4233-4237.

49. Dalle-Donne I, Rossi R, Giustarini D, Milzani A, Colombo R. Protein carbonyl groups as biomarkers of oxidative stress. Clin Chim Acta. 2003;329(1-2):23-38.

50. Halliwell B, Chirico S. Lipid peroxidation: its mechanism, measurement, and significance. $A m$ J Clin Nutr. 1993;57(5 Suppl):715S-724S.

51. Kaye JA, DeCarli C, Luxenberg JS, Rapoport SI. The significance of age-related enlargement of the cerebral ventricles in healthy men and women measured by quantitative computed X-ray tomography. JAm Geriatr Soc. 1992;40(3):225-231.
52. Raupach B, Peuschel SK, Monack DM, Zychlinsky A. Caspase-1-mediated activation of interleukin-1beta (IL-1beta) and IL-18 contributes to innate immune defenses against Salmonella enterica serovar Typhimurium infection. Infect Immun. 2006;74(8):4922-4926.

53. Baranov SV, et al. Mitochondria modulate programmed neuritic retraction. Proc Natl Acad Sci USA. 2019;116(2):650-659.

54. O’Brien LC, Keeney PM, Bennett JP. Differentiation of human neural stem cells into motor neurons stimulates mitochondrial biogenesis and decreases glycolytic flux. Stem Cells Dev. 2015;24(17):1984-1994.

55. Khairova RA, Machado-Vieira R, Du J, Manji HK. A potential role for pro-inflammatory cytokines in regulating synaptic plasticity in major depressive disorder. Int J Neuropsychopharmacol. 2009;12(4):561-578.

56. Hashiguchi K, Zhang-Akiyama QM. Establishment of human cell lines lacking mitochondrial DNA. Methods Mol Biol. 2009;554:383-391.

57. Trettel F, et al. Dominant phenotypes produced by the HD mutation in STHdh(Q111) striatal cells. Hum Mol Genet. 2000;9(19):2799-2809.

58. Vincent J, et al. Small molecule inhibition of cGAS reduces interferon expression in primary macrophages from autoimmune mice. Nat Com mun. 2017;8(1):750.

59. Zhou W, et al. Structure of the human cGAS-DNA complex reveals enhanced control of immune surveillance. Cell. 2018;174(2):300-311.e11.

60. Suofu Y, et al. Dual role of mitochondria in producing melatonin and driving GPCR signaling to block cytochrome c release. Proc Natl Acad Sci USA. 2017;114(38):E7997-E8006.

61. Wang X, et al. Methazolamide and melatonin inhibit mitochondrial cytochrome $\mathrm{C}$ release and are neuroprotective in experimental models of ischemic injury. Stroke. 2009;40(5):1877-1885.

62. Zhang Y, et al. Melatonin inhibits the caspase-1/ cytochrome c/caspase-3 cell death pathway, inhibits MT1 receptor loss and delays disease progression in a mouse model of amyotrophic lateral sclerosis. Neurobiol Dis. 2013;55:26-35.

63. Cardinali DP. Melatonin: clinical perspectives in neurodegeneration. Front Endocrinol (Lausanne). 2019;10:480.

64. Haag SM, et al. Targeting STING with covalent small-molecule inhibitors. Nature. 2018;559(7713):269-273.

65. Yano H, et al. Inhibition of mitochondrial protein import by mutant huntingtin. Nat Neurosci. 2014;17(6):822-831.

66. Jauhari A, Singh T, Singh P, Parmar D, Yadav S. Regulation of miR-34 family in neuronal development. Mol Neurobiol. 2018;55(2):936-945.

67. Stack EC, et al. Chronology of behavioral symptoms and neuropathological sequela in R6/2 Huntington's disease transgenic mice. JComp Neurol. 2005;490(4):354-370.

68. Wang AQ, Wei BP, Zhang Y, Wang YJ, Xu L, Lan K. An ultra-high sensitive bioanalytical method for plasma melatonin by liquid chromatography-tandem mass spectrometry using water as calibration matrix. J Chromatogr B Analyt Technol Biomed Life Sci. 2011;879(23):2259-2264. 